\title{
Scene-based correction of image sensor deficiencies
}

\author{
Master's thesis in image processing at \\ Linköping Institute of Technology by \\ Petter Torle
}

LiTH-ISY-EX-3350-2003

\begin{abstract}
This thesis describes and evaluates a number of algorithms for reducing fixed pattern noise in image sequences by analyzing the observed scene. Fixed pattern noise is the dominant noise component for many infrared detector systems, perceived as a superimposed pattern that is approximately constant for all image frames.

Primarily, methods based on estimation of the movement between individual image frames are studied. Using scene-matching techniques, global motion between frames can be successfully registered with sub-pixel accuracy. This allows each scene pixel to be traced along a path of individual detector elements. Assuming a static scene, differences in pixel intensities are caused by fixed pattern noise that can be estimated and removed.

The algorithms have been tested by using real image data from existing infrared imaging systems with good results. The tests include both a two-dimensional focal plane array detector and a linear scanning one-dimensional detector, in different scene conditions.
\end{abstract}

Keywords: Nonuniformity correction, fixed pattern noise, scenebased, motion estimation, sequence, registration.

\section{Supervisors:}

Ingmar Andersson and Leif Haglund, Saab Bofors Dynamics AB

Examiner:

Klas Nordberg

Linköping, May 6, 2003 



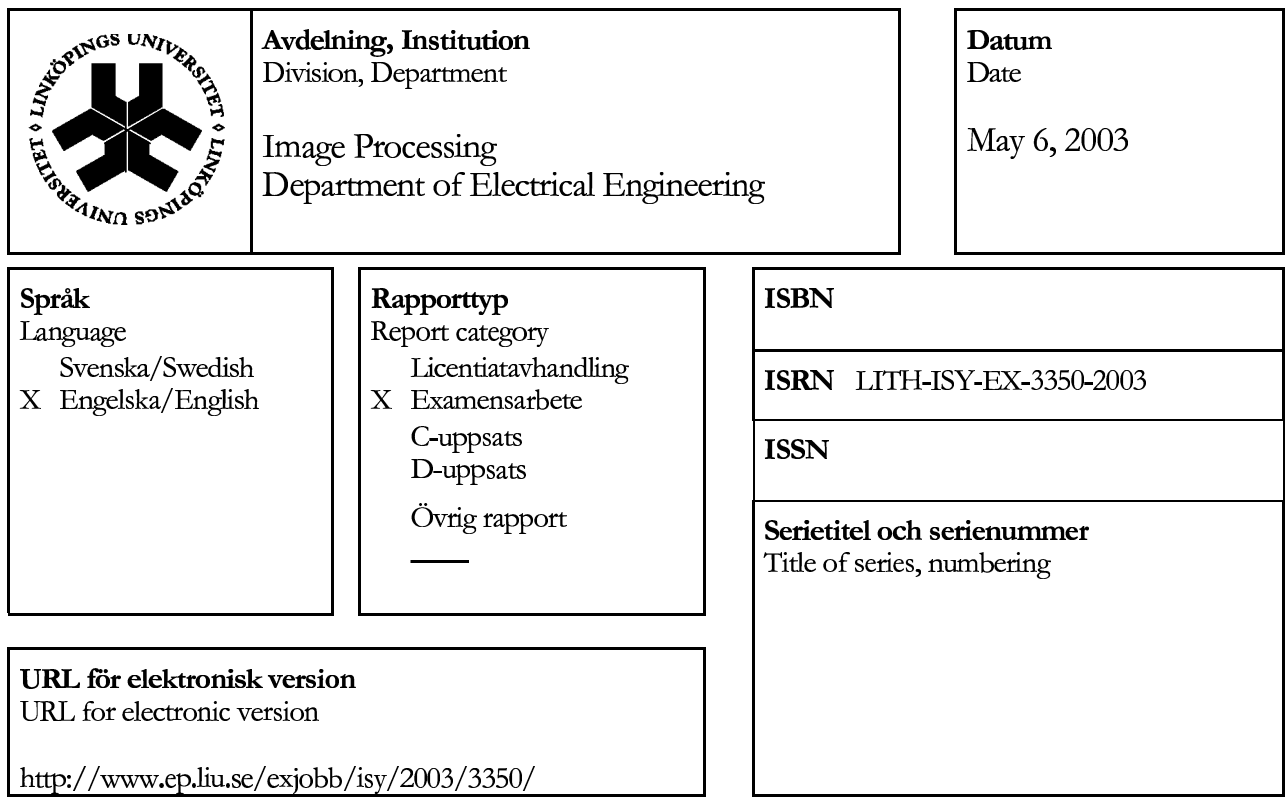

\section{Titel Scenbaserad korrigering av sensordefekter $\mathrm{i}$ bildalstrande sensorer}

Title Scene-based correction of image sensor deficiencies

Författare Petter Torle

Author

\section{Sammanfattning}

Abstract

This thesis describes and evaluates a number of algorithms for reducing fixed pattern noise in image sequences. Fixed pattern noise is the dominant noise component for many infrared detector systems, perceived as a superimposed pattern that is approximately constant for all image frames.

Primarily, methods based on estimation of the movement between individual image frames are studied. Using scene-matching techniques, global motion between frames can be successfully registered with sub-pixel accuracy. This allows each scene pixel to be traced along a path of individual detector elements. Assuming a static scene, differences in pixel intensities are caused by fixed pattern noise that can be estimated and removed.

The algorithms have been tested by using real image data from existing infrared imaging systems with good results. The tests include both a two-dimensional focal plane array detector and a linear scanning onedimensional detector, in different scene conditions.

\section{Nyckelord}

Keywords

Nonuniformity correction, NUC, fixed pattern noise, FPN, scene-based, motion estimation, sequence, registration, IR. 



\section{Acknowledgments}

This master's thesis was done at Saab Bofors Dynamics, Linköping. I would like to thank the following people:

- The initiators to the thesis and my supervisors at Saab Bofors Dynamics, Ingmar Andersson and Leif Haglund

- Mikael Lindgren and Lars Peterson at Saab Bofors Dynamics, Göteborg, who helped me record most of the test image sequences

- My examiner Klas Nordberg at the Department of Electrical Engineering, Linköpings Universitet

- The staff at the section for image processing at Saab Bofors Dynamics

- My opponent Martin Ohlson. 



\section{Abbreviations}

The following abbreviations are used in this document:

FPN $\quad$ Fixed Pattern Noise

IR Infrared

IIR Infinite Impulse Response

IRST Infrared Search and Track

MCA Motion Compensated Average

MSM Multispectral Measurement System

NUC Nonuniformity Correction

QWIP Quantum Well Infrared Photodetector

SNR Signal to Noise Ratio 



\section{Contents}

1 Introduction $\quad 13$

1.1 Background . . . . . . . . . . . . . . . . . . . 13

1.2 Goal of the thesis . . . . . . . . . . . . . . . . 14

1.3 Guidelines and assumptions . . . . . . . . . . . . . 14

1.4 Previous work . . . . . . . . . . . . . . . . 14

1.5 Implementation . . . . . . . . . . . . . . . . . 14

1.6 Thesis overview . . . . . . . . . . . . . . . . 15

2 Infrared sensor technology 17

2.1 Thermal radiation . . . . . . . . . . . . . 17

2.2 Sensor design . . . . . . . . . . . . . . . . . . . 18

2.3 Fixed pattern noise . . . . . . . . . . . . . . . . . 18

2.4 Sensor systems $\ldots \ldots \ldots \ldots$

3 Nonuniformity correction $\quad 23$

3.1 Removing fixed pattern noise . . . . . . . . . . 23

3.2 Basic scene-based nonuniformity correction . . . . . 24

3.3 Ghosting artifacts . . . . . . . . . . . 26

3.4 One-image correction . . . . . . . . . . . . 26

3.5 Enhanced scene-based algorithms . . . . . . . . . 28

4 Estimating image motion 31

4.1 A Fourier-based method . . . . . . . . . . . . . 31

4.2 Sub-pixel accuracy . . . . . . . . . . . . . 33

4.3 Two-component motion estimation . . . . . . . . . 34

4.4 Special case: Fixed pattern noise . . . . . . . . . 37

4.5 Evaluation . . . . . . . . . . . . . . . 37

4.6 Comments on implementation . . . . . . . . . . . 38 
5 Registration-based nonuniformity correction 41

5.1 Motion compensating methods . . . . . . . . . . . 41

5.2 Crossing path . . . . . . . . . . . . . . 43

6 Evaluation $\quad 53$

6.1 Test sequences . . . . . . . . . . . . . . 53

6.2 Evaluation methods . . . . . . . . . . . . . 53

6.3 Temporal noise . . . . . . . . . . . . . . . 53

6.4 One-image correction . . . . . . . . . . . . . . 54

6.5 Temporal highpass filter . . . . . . . . . . . . 55

6.6 Constant statistics . . . . . . . . . . . . . 55

6.7 Motion compensated average . . . . . . . . . . 56

6.8 Crossing path . . . . . . . . . . . . . . . . . 59

7 Results $\quad 63$

7.1 Computational speed . . . . . . . . . . . . . . . 63

7.2 Reference images . . . . . . . . . . . . . . . . . . 64

7.3 Introduced noise . . . . . . . . . . . . . . 65

7.4 Synthetic sequences . . . . . . . . . . . 65

7.5 Visual inspection . . . . . . . . . . . . . . 67

7.6 One-dimensional sensor _ . . . . . . . . . . . . . . . 69

7.7 Example results . . . . . . . . . . . . . . 71

8 Conclusions $\quad \mathbf{7 9}$

8.1 Summary . . . . . . . . . . . . . . . . . 79

8.2 Suggestions for further work . . . . . . . . . . 80 


\section{Chapter 1}

\section{Introduction}

\subsection{Background}

The performance of any image processing system depends on the quality of the input images, which makes image enhancement and pre-processing an important field of research. Compensating for nonlinear sensor optics, enhancing or suppressing different parts of the image, and reducing disturbing noise are examples of common image pre-processing. This thesis concerns the latter.

All image sensors consisting of an array of detector elements suffer more or less from an undesired effect called fixed pattern noise, FPN. This type of noise is caused by the fact that individual detector elements respond differently to incoming irradiance, which is perceived in images as a superimposed pattern, approximately constant from frame to frame. Although the observed image can be quite severely distorted, the fact that the same distortion is present in several acquired images should make it possible to accurately extract the true scene, a process referred to as non-uniformity correction, NUC. Traditionally, this is performed by using hardware temperature references for calibration. Another approach is scene-based techniques, where information from the observed scene itself is used to reduce fixed pattern noise. For example, two different detector elements that observe the same part of the scene should produce the same output. If not, correction parameters can be slightly adjusted to compensate.

For infrared (IR) imaging systems, fixed pattern noise is usually the dominant noise component. It is also depending on scene irradiance, detector temperature and is slightly time varying, which makes it difficult to correct this problem with an initial factory calibration. 


\subsection{Goal of the thesis}

The goal of this thesis is to design and evaluate scene-based nonuniformity correction algorithms that are able to suppress fixed pattern noise without need for external hardware such as temperature reference equipment. In particular, algorithms should be able to accurately estimate motion between images and use this knowledge to improve performance.

\subsection{Guidelines and assumptions}

The correction algorithms should be developed with a future real-time hardware implementation in mind. This means for example that the resulting output must not depend on any future input signals, and that the execution time for each input frame should be approximately constant. Furthermore, the input images are assumed to be captured from a static scene, and the global motion between frames is assumed to consist only of translations in the image plane. The methods should be designed both for a two-dimensional array detector and a one-dimensional scanning detector.

\subsection{Previous work}

Methods for scene-based removal of fixed pattern noise have been researched for some time. Yet, relatively few different correction methods were found when a search for nonuniformity correction related articles was conducted. The most widely used algorithms $[4,2,3,10]$ are either based on making each detector output similar to those of the surrounding detectors in the array, or based on statistical analysis. Some more advanced methods depending on image motion estimation was also found $[7,8]$.

\subsection{Implementation}

The nonuniformity correction algorithms are evaluated using both synthetic and real image data. Image sequences have been recorded at Saab Bofors Dynamics in Göteborg as well as in Linköping. Algorithms and utilities have been developed in a MATLAB environment. 


\subsection{Thesis overview}

Chapter 2 - Infrared sensor technology

This chapter gives a short introduction to infrared sensor technology, including a discussion on some of the factors contributing to fixed pattern noise in IR images. Basic properties of the sensors used to acquire IR images for this thesis are also mentioned.

Chapter 3 - Nonuniformity correction

In this chapter, different nonuniformity correction methods are categorized and some basic scene-based algorithms are presented.

Chapter 4 - Estimating image motion

For more advanced nonuniformity correction, accurate knowledge of the global image motion is required. A Fourier-based method for motion estimation is described and evaluated.

Chapter 5 - Registration-based nonuniformity correction

In this chapter, nonuniformity correction methods relying on knowledge of image motion are presented.

\section{Chapter 6 - Evaluation}

The proposed algorithms are evaluated individually in this chapter, using several test sequences. The quality of the resulting output data is discussed, and some methods are modified to perform better.

Chapter 7 - Results

The results from the previous chapter are summed up and the different correction methods are compared in various ways.

Chapter 8 - Conclusions

This chapter concludes the thesis, with comments on the results and suggestions for further work. 



\section{Chapter 2}

\section{Infrared sensor technology}

\subsection{Thermal radiation}

Any given object emits thermal electromagnetic radiation, distributed over an entire spectrum of wavelengths. Figure 2.1 shows emittance spectrums for the temperatures $T=300,400$ and $500 \mathrm{~K}$.

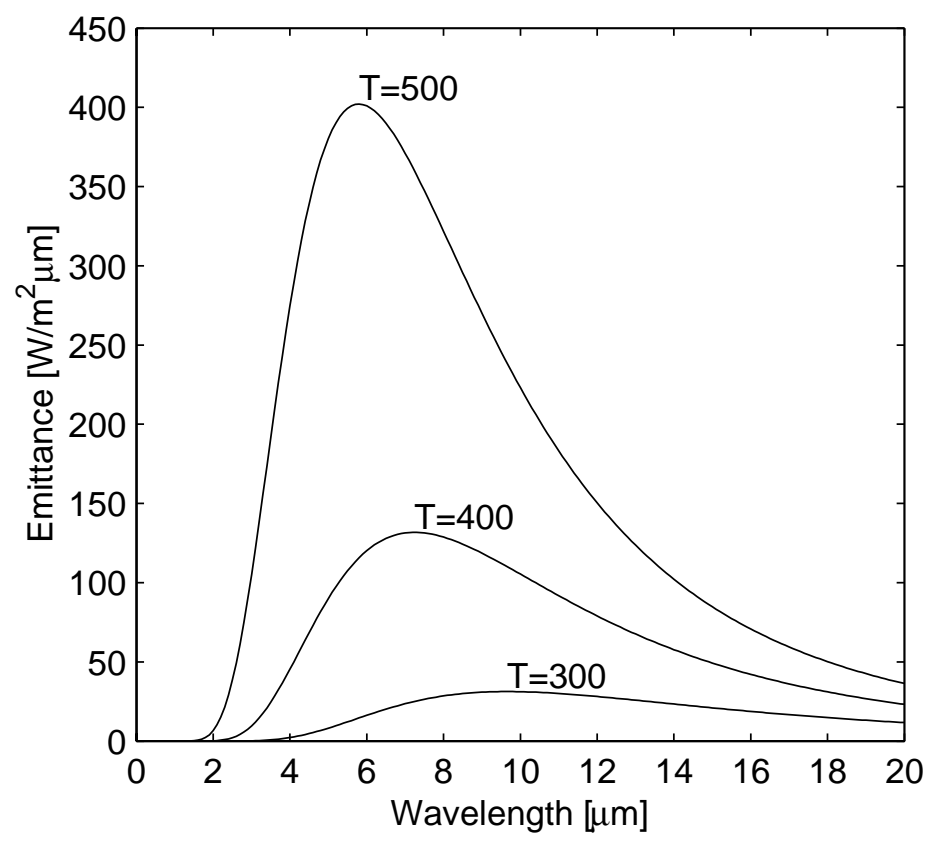

Figure 2.1: Thermal radiation for different temperatures. 
The radiation for a specific temperature $T$ has an intensity peak at the wavelength given by Wien's displacement law:

$$
\lambda_{\max }=\frac{k}{T} \quad, \quad k=2.898 \cdot 10^{-3}
$$

If the temperature is high enough, the radiation can be perceived by the human eye as colored light. However, most natural objects have a much lower temperature, corresponding to an intensity peak in the 0.8-30 $\mu \mathrm{m}$ wave band. These wavelengths are part of the infrared band, positioned between visible light and microwaves in the electromagnetic spectrum. Sensors that detect photons with wavelengths in this range thus produce an image with intensities corresponding to the temperatures of the observed scene.

\subsection{Sensor design}

A widely used detector design is a focal plane array of photodiode elements, made of mercury-cadmium-telluride. For a two-dimensional array, each element represents one pixel in the output image. The array can also be one-dimensional, in which case the array needs to undergo a scanning motion to generate an entire image.

\subsection{Fixed pattern noise}

Individual elements in the detector array differ in responsivity to incoming irradiance, which is the main reason why fixed pattern noise occur in an image. Some pixels end up too bright, some too dark, depending on a multitude of parameters [1]. Some of these parameters may be identified in advance and compensated for, but it is impossible to compensate for all. The result is an image with a superimposed pattern that varies with unknown environmental parameters, leading to a time varying fixed pattern noise.

The most common fixed pattern noise sources include:

\section{Fabrication errors}

Inaccuracies in the fabrication process give rise to variations in geometry and substrate doping quality of the detector elements. This leads directly to offset and gain variations across the detector array. 


\section{Cooling system}

In order to deliver any useful data, many sensors must be cooled to an operating temperature below $100 \mathrm{~K}$. Small deviations in the regulated temperature are hard to avoid, and may have large impact on detector parameters.

\section{Electronics}

For detector arrays, variations in the read-out electronics is a common source of fixed pattern noise, often visible as grid and line patterns.

System $\frac{1}{f}$ noise

Apart from white noise, the power spectrum of typical temporal noise present in infrared image sequences also consists of a component proportional to the inverse of the frequency. The origin

of this $\frac{1}{f}$ component is not completely known, but it causes drift of system parameters, ultimately leading to time-varying fixed pattern noise.

\section{Optics}

Some fixed pattern noise is also caused by the sensor optics. This includes a decrease in signal intensity at the edges of the image and different kinds of circular image artifacts.

\subsection{Sensor systems}

The sensor used to acquire most of the test sequences for this thesis is called MSM, multispectral measurement system. The MSM uses a two-dimensional focal plane array, cooled to a temperature of about $80 \mathrm{~K}$ to minimize disturbance from the sensor chip itself.

To illustrate the level of nonuniformity for the MSM system, figure 2.2 shows six raw frames from one of the test sequences. As a preview of things to come, figure 2.3 shows what the corresponding frames may look like when nonuniformity correction has been performed. In this particular case, the resulting parameters after processing all 400 frames of the sequence have been used to calibrate each image.

Compared to the latest IR sensor technology, the MSM is slightly outdated and the produced images are severely distorted by fixed pattern noise. A somewhat more sophisticated detector based on quantum well infrared photodetector (QWIP) technology has also been studied. 


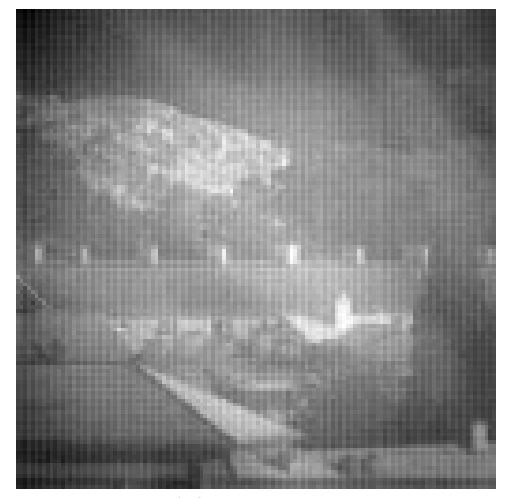

(a) Frame 25

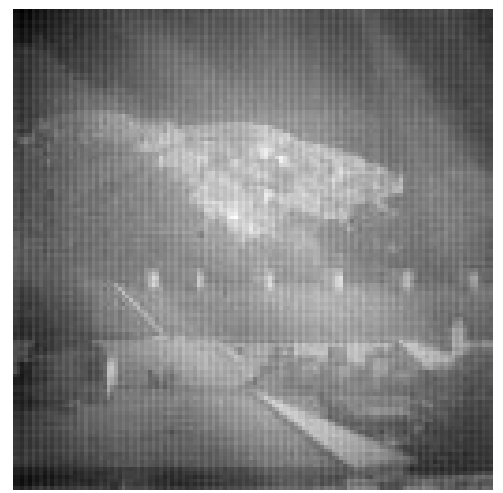

(c) Frame 75

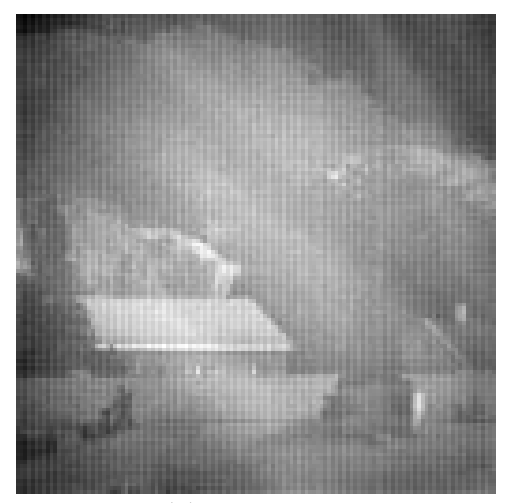

(e) Frame 200

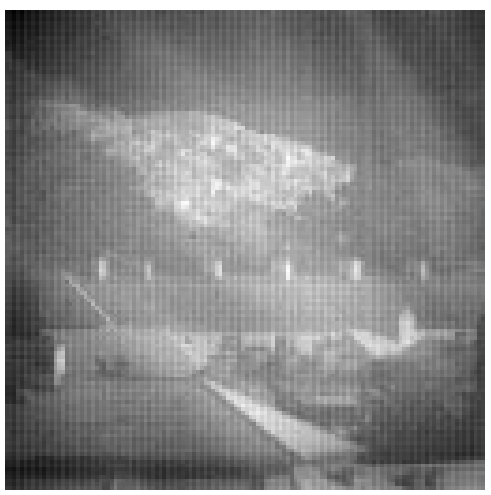

(b) Frame 50

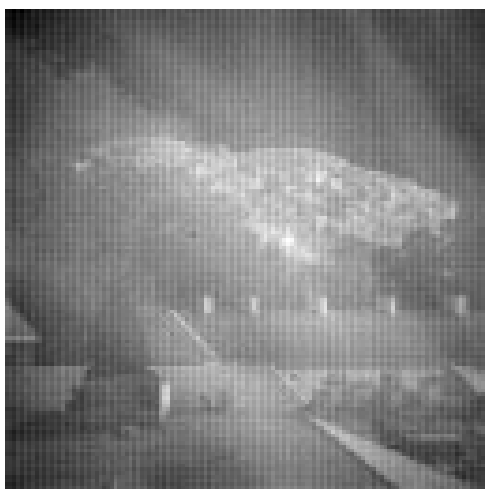

(d) Frame 100

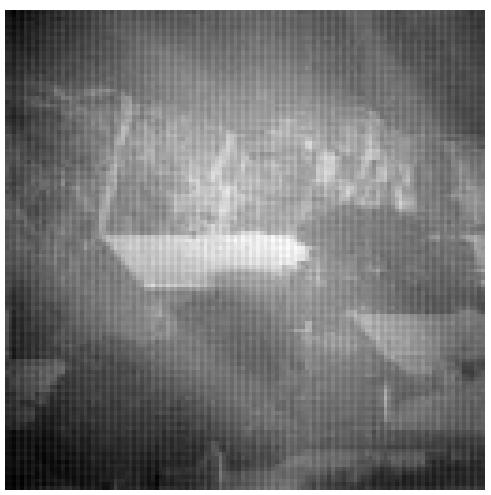

(f) Frame 400

Figure 2.2: Raw sensor images from one of the MSM test sequences. 


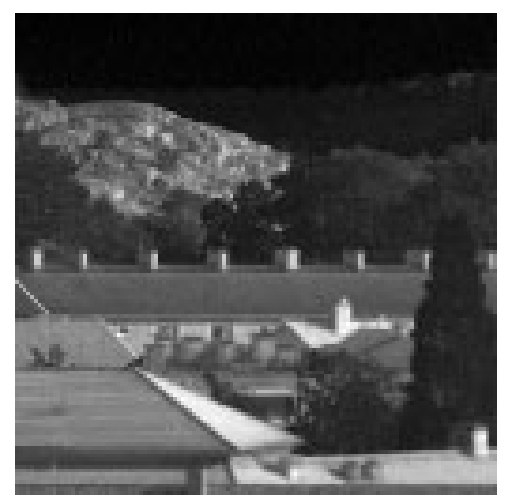

(a) Frame 25

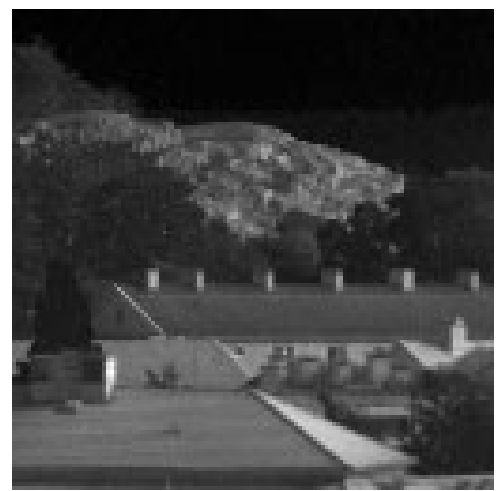

(c) Frame 75

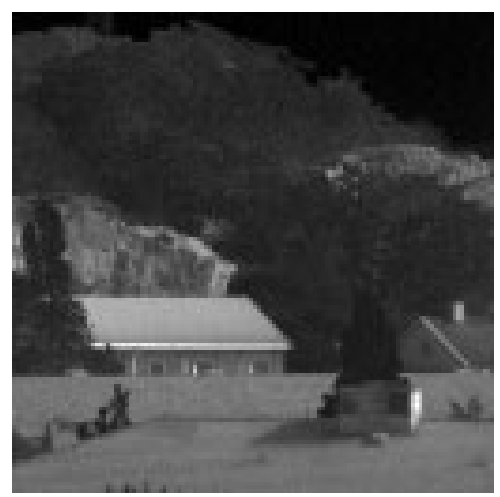

(e) Frame 200

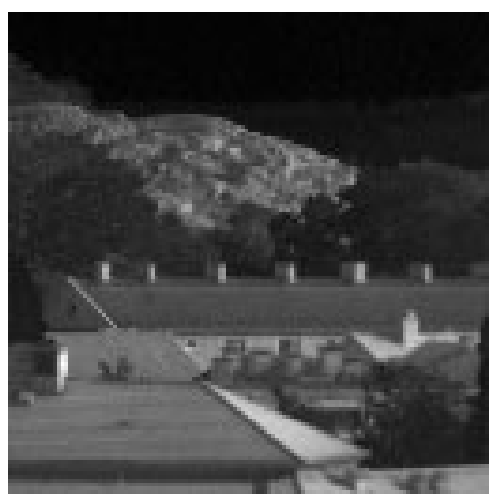

(b) Frame 50

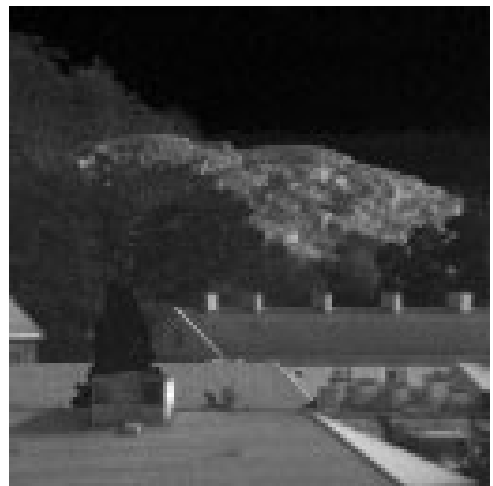

(d) Frame 100

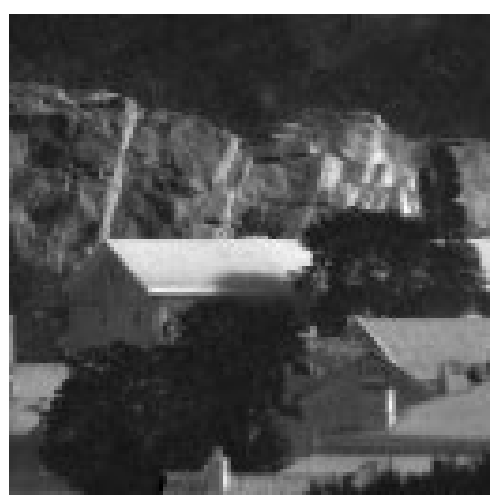

(f) Frame 400

Figure 2.3: Corrected sequence. 
As mentioned earlier, an alternative to a two-dimensional detector array is to use a one-dimensional scanning array. Saab Bofors Dynamics has developed an IRST system (Infrared Search and Track) equipped with such a sensor. The final image is generated by horizontally sweeping the column of detector elements. Fixed pattern noise for this system is then visible as horizontal stripes, which can be regarded as a special case of two-dimensional fixed pattern noise. Raw images from this sensor can be so degraded that it is difficult to discern any scene information at all.

Typical raw images from these sensors are shown in figure 2.4.

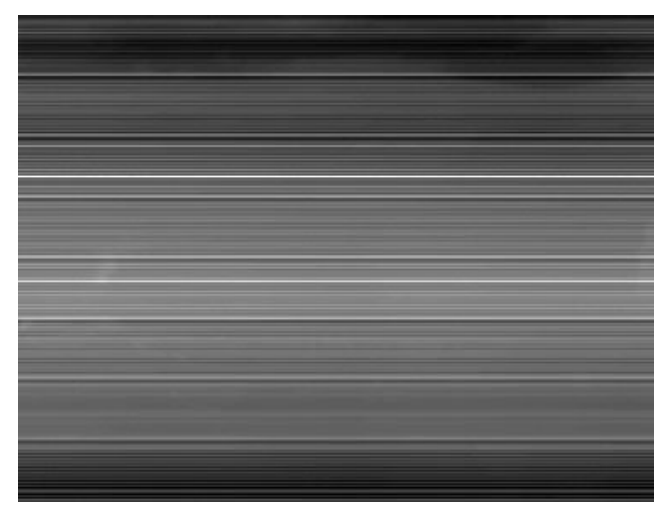

(a) IRST-system, using a horizontally scanning $1 \mathrm{D}$ detector array.

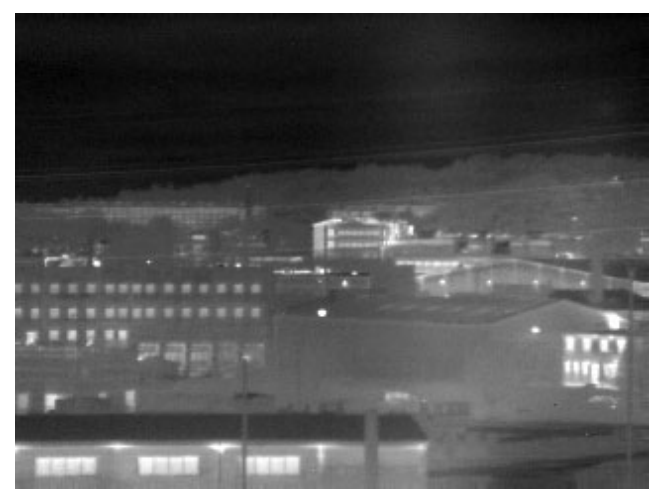

(b) A modern QWIP camera

Figure 2.4: Typical raw IRST and QWIP sensor images. 


\section{Chapter 3}

\section{Nonuniformity correction}

\subsection{Removing fixed pattern noise}

Reducing fixed pattern noise to a minimum is, of course, essential to any imaging system. Many techniques have been developed to perform such nonuniformity correction, most of which are based on a linear irradiance-voltage model:

$$
x=z \cdot g+o
$$

where the true scene value $z$ is scaled by a gain factor $g$ and offset by an offset term $o$ to produce the observed detector output $x$. It is also common to model the fixed pattern noise completely as an offset shift:

$$
x=z+o
$$

The correction parameters $g$ and $o$ are especially straight forward to obtain if a true scene value $z$ is known. This can be achieved by exposing the sensor to a surface with known uniform temperature. Using more than one reference temperature, both gain and offset correction parameters can be resolved. This approach is a widely used reference-based method for nonuniformity correction. It is also possible to use only one uniform temperature surface, imaged with two different integration times. The MSM system, used to acquire infrared image sequences in this thesis, can use both these calibration methods [1]. Figure 3.1 shows typical MSM gain and offset correction parameters, obtained when applying reference based calibration. 


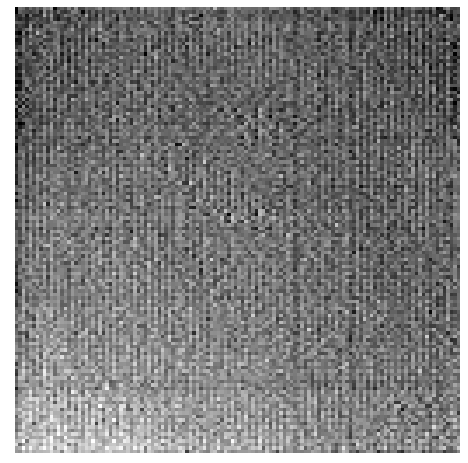

(a) Offset

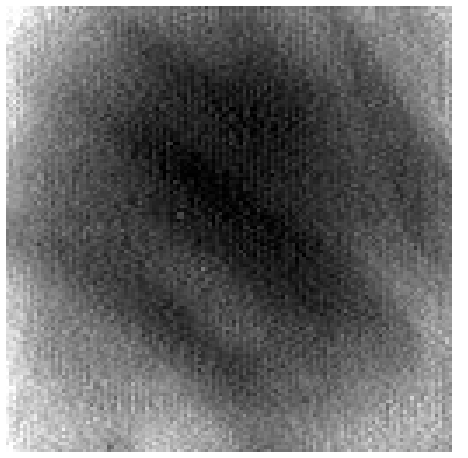

(b) Gain

Figure 3.1: Typical MSM correction parameters.

\subsection{Basic scene-based nonuniformity correc- tion}

Though imaging systems with reference-based nonuniformity correction are accurate, they often involve opto-mechanic components and temperature references that are expensive and complex in design. To overcome this problem, a lot of research has been focused on performing sensor calibration entirely in software. Several methods have been presented where the observed scene itself is used to calibrate the detector elements - scene-based nonuniformity correction.

In this section, two scene-based correction methods are presented: temporal highpass filter[2,3] and constant statistics[4]. Both are well known and appears frequently in the literature.

\section{Temporal highpass filter}

Images from a sequence of infrared image data consist of scene information, varying from frame to frame, and a fixed pattern noise, roughly the same in all frames. This means that when studying each pixel individually over time, high-frequency information belongs to the scene, while low-frequency information belongs to fixed pattern noise. An estimate of the noise is thus obtained by lowpass filtering the image sequence along the temporal axis. When subtracting this estimate from an input image frame, nonuniformity correction is 
performed. The whole process acts like a temporal highpass filter, as shown in figure 3.2. A temporal average $f$ of the image sequence is generated by a recursive IIR filter and subtracted from the current frame. The output image at time index $n$ becomes

$$
y_{n}=x_{n}-f_{n}
$$

where

$$
f_{n}=\frac{x_{n}+(n-1) f_{n-1}}{n}
$$

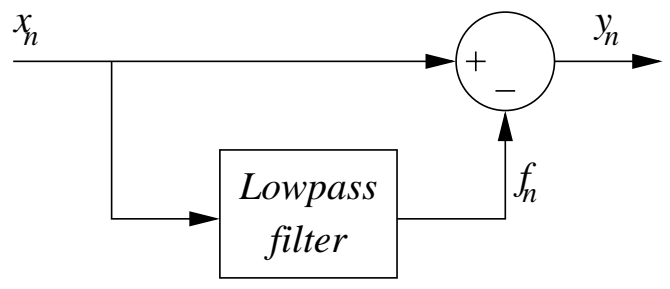

Figure 3.2: Temporal highpass filter.

\section{Constant statistics}

The constant statistics algorithm is similar to the temporal highpass filter but is extended to estimate both gain and offset parameters. The assumptions of this method are that the temporal means and variances are identical for all pixels. For this assumption to hold, it is necessary that over time, all possible scene irradiance values will be observed by all detector elements. This means that image motion must exist, either from a dynamic scene or from movement of the sensor. For the linear model (3.1), the temporal mean value is

$$
m_{x}=\mathrm{E}[x]=\mathrm{E}[z \cdot g+o]=g \cdot \mathrm{E}[z]+o=g \cdot m_{z}+o
$$

and the mean deviation is

$$
s_{x}=\mathrm{E}\left[\left|x-m_{x}\right|\right]=\mathrm{E}\left[\left|g \cdot\left(z-m_{z}\right)\right|\right]=g \cdot s_{z}
$$

It is further assumed that the temporal statistics of $z$ is constant for all detector elements. For example, $z$ can be assumed to have zero mean and unity mean deviation $\left(m_{z}=0, s_{z}=1\right)$. This can be done without losing generality since the true values of $m_{z}$ and $s_{z}$ 
can be incorporated into the parameters $o$ and $g$ respectively. The expressions are now written

$$
\begin{aligned}
m_{x} & =o \\
s_{x} & =g
\end{aligned}
$$

which allows solving (3.1) as:

$$
z=\frac{x-m_{x}}{s_{x}}
$$

Note that this expression equals the temporal highpass filter of the previous section when neglecting the denominator.

The estimated mean of $x$ can be calculated recursively like before (3.3), while a recursive equation for $s_{x}$ is given by

$$
s_{x, n}=\frac{\left|x_{n}-m_{x, n}\right|+(n-1) s_{x, n-1}}{n}
$$

\subsection{Ghosting artifacts}

If some part of the scene remains motionless for several algorithm iterations, it will be considered to be fixed pattern noise and as such be blended into the background. When this part eventually resumes motion, it will leave an inverse ghost image in its place. This ghosting artifact is a problem to most scene-based nonuniformity correction algorithms. Ghosting may occur even when the image motion is sufficient. For example, a view of the horizon may generate a ghost image when panning the sensor horizontally.

A simple method for reducing the ghosting effect is to prohibit parameter updates if the magnitude of the change at each pixel is smaller than a fixed threshold. [5]

\subsection{One-image correction}

Usually, some properties and characteristics of the fixed pattern noise is known in advance. For example, pixel intensity is generally lower at the edges of the image, and pixel-to-pixel correlation is visible as grid and line patterns. This knowledge can be used to produce an estimate of the fixed pattern noise as an initial set of correction parameters for further processing by the nonuniformity correction algorithms.

Figure 3.3(a) shows a close-up of an IR image, with grid noise typical for sensors with detector arrays. It seems that every second 
pixel is somewhat correlated. This is also evident when examining the Fourier transform of the image, figure 3.3(b), where the two bright pixels located in the middle of the top and left edges correspond to a sine-shaped signal with a wavelength of 2 pixels.

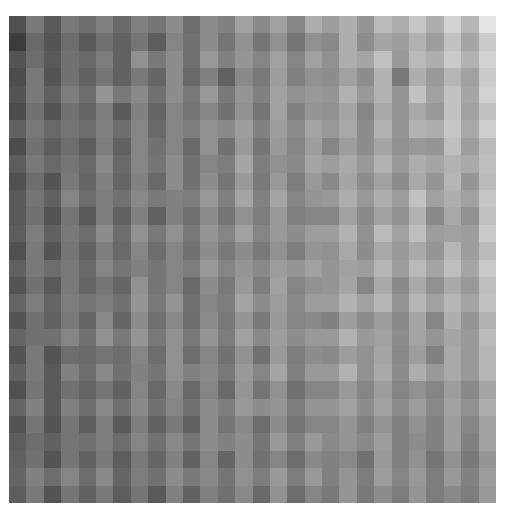

(a) Grid noise

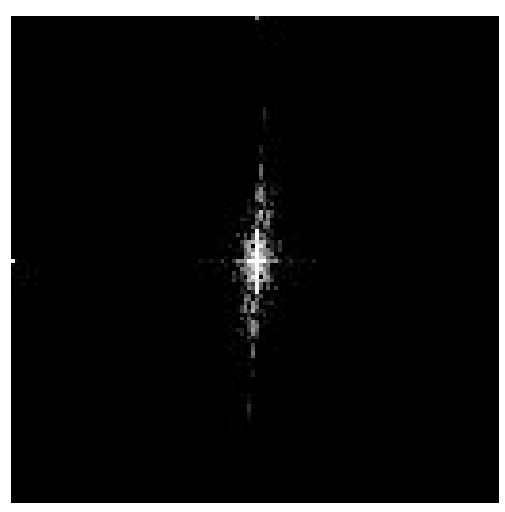

(b) Fourier transform

Figure 3.3: IR image properties

Once identified, these frequency components may be filtered out to remove most of the grid noise, as shown in figure 3.4.

With the typical correction parameters of figure 3.1 in mind, the grid noise is mostly additive in origin, while the pixel intensity decrease at the image edges is part of a slowly varying multiplicative fixed pattern noise.

By taking the logarithm of the image, this noise will be turned into additive noise, which then can be reduced by applying a high pass filter. This is sometimes referred to as homomorph filtering [9]. In figure 3.5, this filter is applied to the previous result from figure 3.4(b).

Some artifacts are still clearly visible in the image. The sharp vertical lines are always located at the same position, probably caused by the read-out electronics. The lines can possibly be reduced by modifying the grid noise filter or adding a mask manually, but it would be wise not to make the initial correction procedure too sensorspecific. 


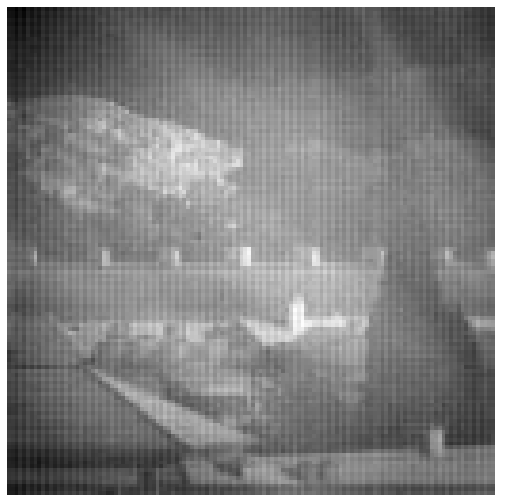

(a) Original image

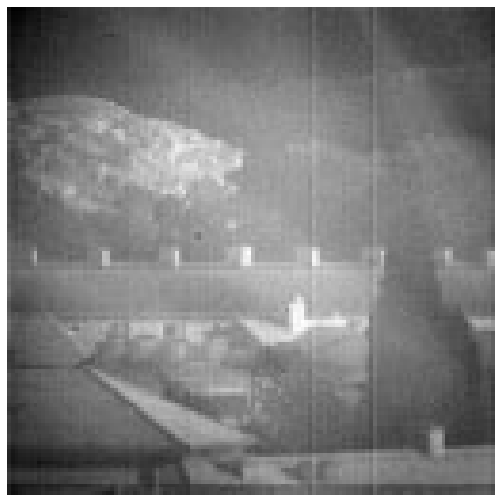

(b) Filtered image

Figure 3.4: One-image correction of grid noise



(a) Original image

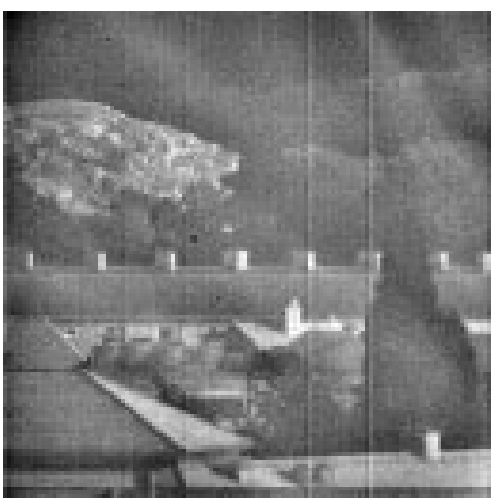

(b) Filtered image

Figure 3.5: Homomorph filtering

\subsection{Enhanced scene-based algorithms}

The nonuniformity correction methods described so far assume that motion between the image frames exists, but no further analysis of this motion is performed. It is reasonable that performance would increase if knowledge of the actual image movement is incorporated 
into the algorithms. This leads to a higher level of scene-based algorithms, referred to as registration-based nonuniformity correction. With these methods, computational complexity and computer memory demands are significantly increased.

Being able to accurately estimate the image motion is naturally an important part of registration-based algorithms. Such a motion estimation algorithm will be described in the following chapter. 



\section{Chapter 4}

\section{Estimating image motion}

The performance of many nonuniformity correction algorithms depends heavily on an accurate estimate of the global motion between image frames. In this thesis, the motion is assumed to consist only of translation, neglecting any scaling, rotation or other warping of the images. A well known motion estimation method based on the Fourier phase of the images [6] is presented in this chapter.

\subsection{A Fourier-based method}

Consider two images, $f$ and $g$, related to each other by a translation vector $\mathbf{x}_{\mathbf{0}}$ as in $g(\mathbf{x})=f\left(\mathbf{x}-\mathbf{x}_{\mathbf{0}}\right)$. The Fourier shift theorem then states that the corresponding Fourier transforms $F$ and $G$ are related as:

$$
G(\boldsymbol{\omega})=F(\boldsymbol{\omega}) \cdot e^{-i 2 \pi \boldsymbol{\omega} \mathbf{x}_{0}}
$$

Now let

$$
H(\boldsymbol{\omega}) \equiv \frac{G(\boldsymbol{\omega})}{F(\boldsymbol{\omega})}=e^{-i 2 \pi \boldsymbol{\omega} \mathbf{x}_{0}}
$$

The inverse Fourier transform of $H$ is given by

$$
h(\mathbf{x})=\delta\left(\mathbf{x}-\mathbf{x}_{\mathbf{0}}\right)
$$

The dirac function is recognized in the image $h$ as an intensity peak, which can be found by simply scanning for the global maximum value. The coordinate of this peak corresponds directly to the translation vector $\mathbf{x}_{\mathbf{0}}$. The procedure is presented in figure 4.1. The second Lenna image is shifted 6.0 pixels vertically and 2.2 pixels horizontally in respect to the first image. The resulting intensity peak can be 
seen clearly in image $4.1(\mathrm{c})$, with coordinates corresponding to the translation vector.

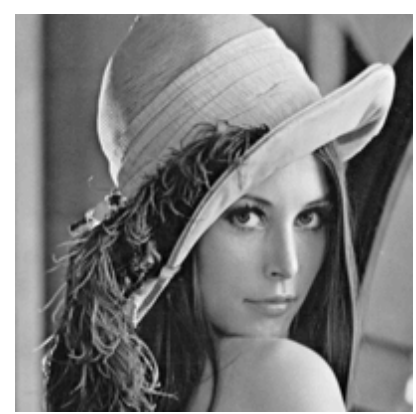

(a) Lenna image 1

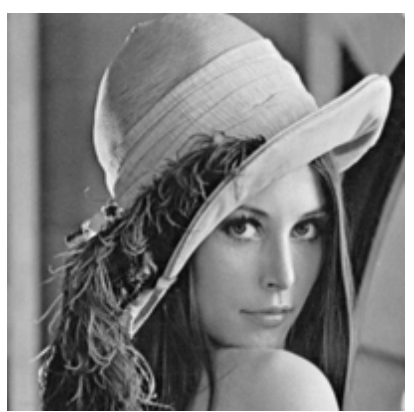

(b) Lenna image 2

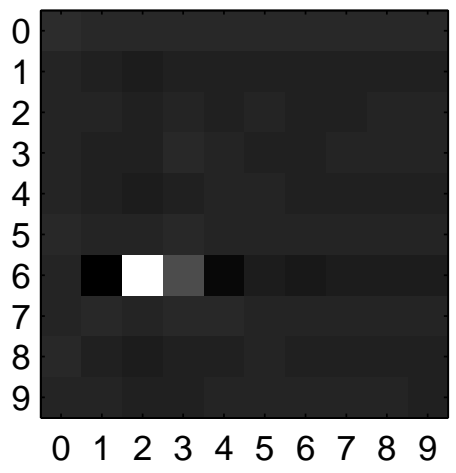

(c) $h(\mathbf{x})$

Figure 4.1: Image motion estimation. Image 2 has been shifted 6.0 pixels vertically and 2.2 pixels horizontally in respect to image 1 .

Note that these calculations assume that there is a one-to-one mapping of pixels in the two images. This is of course not true. Some new scene data has inevitably been introduced at the edges of the image, while other has been translated out of the image frame and lost. These irregularities cause noise that affect the estimation accuracy. To overcome this problem, the magnitude of pixels at the edges of the images can be decreased by spatially filtering the two images with a suitable window function. 


\subsection{Sub-pixel accuracy}

The translation estimates delivered by the proposed method are integer values. Generally, however, sub-pixel accuracy is required. This can be achieved in a number of ways. For example, finding the maximum of a second-degree polynomial surface fitted to the region around the intensity peak increases estimation accuracy by several tenths of a pixel. This solution has its drawbacks though. Figure 4.2 shows the translation estimates of an image shifted one pixel in steps of 0.1 pixels. It seems that the translation estimates are weighted towards integer values. A reason for this may be that a second-degree surface is not really an optimal model for the intensity peak.

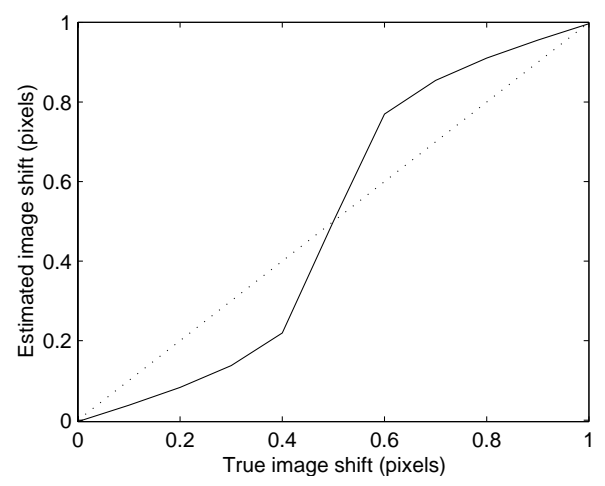

Figure 4.2: Estimates are weighted towards whole pixels.

An alternative method for sub-pixel accuracy is Fourier phase expansion, where the phase of $H$ is multiplied by a scaling constant $C$. Algebraically:

$$
\tilde{H}(\boldsymbol{\omega}) \equiv|H(\boldsymbol{\omega})| \cdot\left(\frac{H(\boldsymbol{\omega})}{|H(\boldsymbol{\omega})|}\right)^{C}
$$

Using $H$ from (4.1):

$$
\tilde{H}(\boldsymbol{\omega})=e^{-i 2 \pi \boldsymbol{\omega} \mathbf{x}_{0} C}
$$

Like before, this image is inverse transformed

$$
\tilde{h}(\mathbf{x})=\delta\left(\mathbf{x}-\mathbf{x}_{\mathbf{0}} C\right)
$$

The position of the intensity peak has now been scaled by the constant $C$, which means that the integer coordinate of the global maximum 
should be divided by $C$ in order to find the translation vector $\mathbf{x}_{\mathbf{0}}$. This procedure effectively increases the estimation accuracy by a factor of $C$. Naturally, this value should be chosen as large as possible. In practice this is limited by the size of the image and noise, which grows larger with increasing scale constant.

The two methods for sub-pixel accuracy can be combined to increase performance even more.

\subsection{Two-component motion estimation}

The described motion estimation method assumes only one dominant image motion in the sequence. In this section it will be shown how the method deals with several motion components, especially a fixed pattern superimposed on moving images.

Consider the case where two patterns undergoing different motions are combined additively in each image. The images $f$ and $g$ can then be written as

$$
\begin{aligned}
& f(\mathbf{x})=a(\mathbf{x})+b(\mathbf{x}) \\
& g(\mathbf{x})=a\left(\mathbf{x}-\mathbf{x}_{\mathbf{a}}\right)+b\left(\mathbf{x}-\mathbf{x}_{\mathbf{b}}\right)
\end{aligned}
$$

where $a$ and $b$ represent two patterns, displaced in the second image by the vectors $\mathbf{x}_{\mathbf{a}}$ and $\mathbf{x}_{\mathbf{b}}$. As before, the images are Fourier transformed and divided:

$$
\begin{aligned}
F(\boldsymbol{\omega}) & =A(\boldsymbol{\omega})+B(\boldsymbol{\omega}) \\
G(\boldsymbol{\omega}) & =A(\boldsymbol{\omega}) \cdot e^{-i 2 \pi \boldsymbol{\omega} \mathbf{x}_{\mathbf{a}}}+B(\boldsymbol{\omega}) \cdot e^{-i 2 \pi \boldsymbol{\omega} \mathbf{x}_{\mathbf{b}}} \\
H(\boldsymbol{\omega})=\frac{G(\boldsymbol{\omega})}{F(\boldsymbol{\omega})} & =\frac{A}{A+B}(\boldsymbol{\omega}) \cdot e^{-i 2 \pi \boldsymbol{\omega} \mathbf{x}_{\mathbf{a}}}+\frac{B}{A+B}(\boldsymbol{\omega}) \cdot e^{-i 2 \pi \boldsymbol{\omega} \mathbf{x}_{\mathbf{b}}}
\end{aligned}
$$

In this case, the image data is not cancelled out. $H$ is now a sum of two shifted terms, each weighted in some sense by the frequency content of the corresponding image in relation to the frequency content of the combined images. Without getting too far into signal theory, it would be reasonable that the Fourier inverse of $H$ now consists of not one, but two intensity peaks. The peaks should be located at $\mathbf{x}_{\mathbf{a}}$ and $\mathbf{x}_{\mathbf{b}}$ with magnitudes related to the corresponding image frequency content. Figure 4.3 shows the results when two motion components are present. Two images of flowers, related by a translation of 4.0 pixels horizontally and 1.0 pixels vertically, have been added to the 
Lenna image pair from figure 4.1. As seen in figure 4.3(c), the image $h(\mathbf{x})$ contains two intensity peaks at the expected locations. The background noise level is also higher.

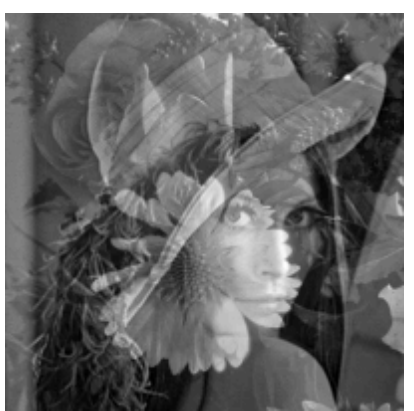

(a) Lenna with flowers 1

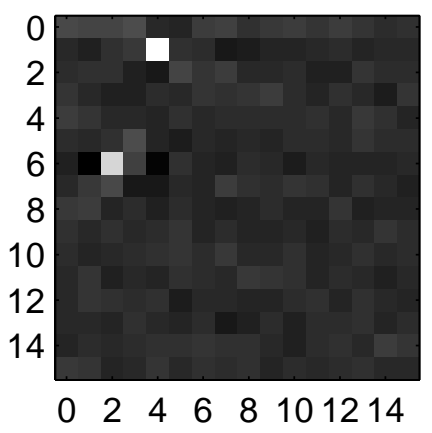

(c) $h(\mathbf{x})$

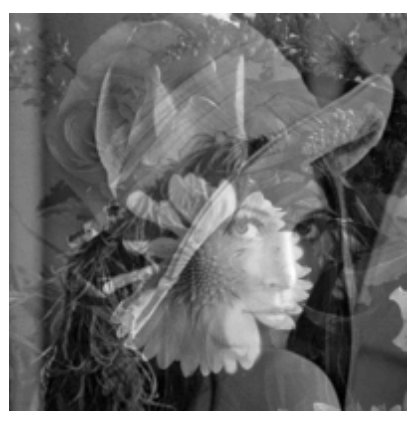

(b) Lenna with flowers 2

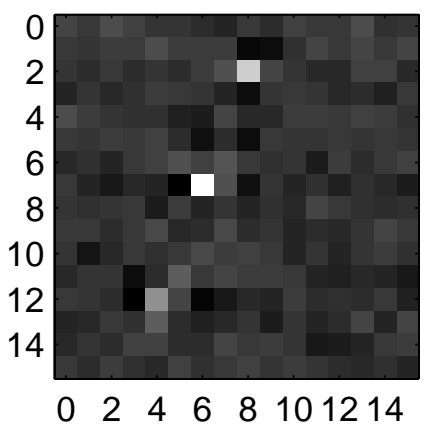

(d) $\tilde{h}(\mathbf{x}), C=2$

Figure 4.3: Two-component motion estimation.

To achieve sub-pixel accuracy as before, the phase of $H$ should be multiplied by a scaling constant (definition 4.2). However, the expression for the normalized $H$ now involves a sum of two exponential functions. Phase-expanding such an expression gives the following 
results. For now, only the exponential functions are considered.

$$
\begin{aligned}
C=2 \quad \tilde{H}(\boldsymbol{\omega}) & \simeq\left(e^{i 2 \pi \boldsymbol{\omega} \mathbf{x}_{\mathbf{a}}}+e^{i 2 \pi \boldsymbol{\omega} \mathbf{x}_{\mathbf{b}}}\right)^{2}= \\
& =e^{i 2 \pi \boldsymbol{\omega} 2 \mathbf{x}_{\mathbf{a}}}+2 e^{i 2 \pi \boldsymbol{\omega}\left(\mathbf{x}_{\mathbf{a}}+\mathbf{x}_{\mathbf{b}}\right)}+e^{i 2 \pi \boldsymbol{\omega} 2 \mathbf{x}_{\mathbf{b}}} \\
C=3 \quad \tilde{H}(\boldsymbol{\omega}) & \simeq\left(e^{i 2 \pi \boldsymbol{\omega} \mathbf{x}_{\mathbf{a}}}+e^{i 2 \pi \boldsymbol{\omega} \mathbf{x}_{\mathbf{b}}}\right)^{3}= \\
& =e^{i 2 \pi \boldsymbol{\omega} 3 \mathbf{x}_{\mathbf{a}}}+3 e^{i 2 \pi \boldsymbol{\omega}\left(2 \mathbf{x}_{\mathbf{a}}+\mathbf{x}_{\mathbf{b}}\right)}+ \\
& +3 e^{i 2 \pi \boldsymbol{\omega}\left(\mathbf{x}_{\mathbf{a}}+2 \mathbf{x}_{\mathbf{b}}\right)}+e^{i 2 \pi \boldsymbol{\omega} 3 \mathbf{x}_{\mathbf{b}}}
\end{aligned}
$$

The two exponential functions have been phase scaled properly, but significant noise has been introduced by the additional terms in the binomial expansion. These terms will all be present as intensity peaks in the resulting inverse transformed image $\tilde{h}(\mathbf{x})$, as shown in figure 4.3(d). The locations of the peaks are determined by the possible combinations of $C$ added original translation vectors, see figure 4.4 for an example.

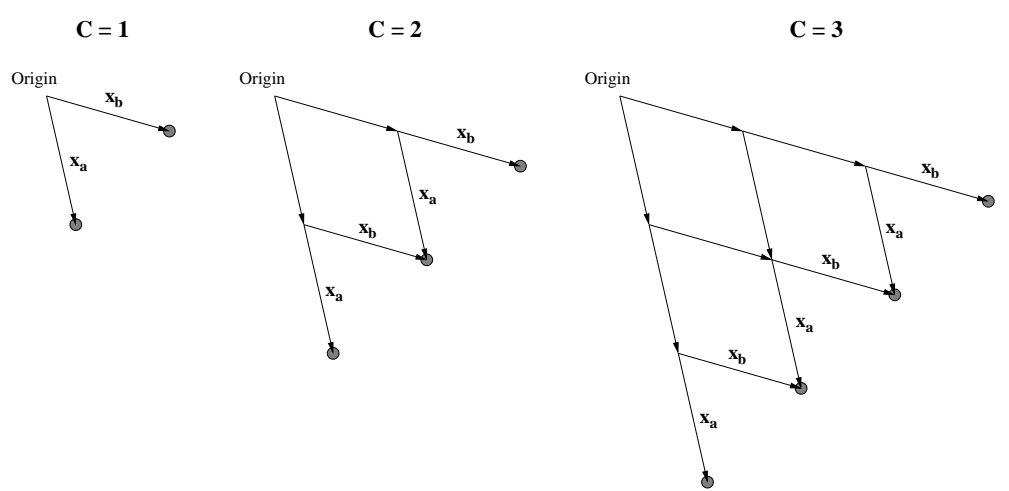

Figure 4.4: Intensity peak positions for two-component image motion with $C=1,2,3$.

Scanning for a global intensity maximum is no longer sufficient for finding the correct translation vectors. However, all the intensity peaks can be found by gathering $C+1$ local maxima. The true peaks are those with the largest in-between distance. Another method is to start without any phase expansion and register the original positions of the intensity peaks. For a given scale factor $C$, new peak positions can be extrapolated and used as centers for smaller regions where a local maximum search is performed. 
So far the frequency information contributed to $H(\boldsymbol{\omega})$ by the remains of the image data $A(\boldsymbol{\omega})$ and $B(\boldsymbol{\omega})$ has been neglected. This noise is unfortunately not insignificant. The magnitude of the intensity peaks in the image $h(\mathbf{x})$ drops rather rapidly with increasing scale factor, and drowns completely in background noise after just a few steps.

\subsection{Special case: Fixed pattern noise}

Image sequences with fixed pattern noise can be considered to be a special case of two-component image motion. Since the noise pattern is motionless, one of the resulting intensity peaks in the image $h(\mathbf{x})$ is always located at the origin. This makes it easier to identify the true image motion.

When used in combination with a nonuniformity correction algorithm, the fixed pattern noise is hopefully reduced with each new frame. The undesired intensity peaks will eventually become less significant, and after some time the image motion consists essentially of only one component.

\subsection{Evaluation}

To evaluate the accuracy of this algorithm in the presence of fixed pattern noise, normal distributed gain and offset coefficients are applied to an 8 bit grayscale image pair like the one in figure 4.1, related by a translation of 2.4 pixels horizontally and 1.8 pixels vertically. The relative translation is then estimated using the described algorithm with $C=2$. The mean absolute error of the estimate is presented for several levels of nonuniformity in figure 4.5.

When the noise becomes too dominant in the image, no relative translation is detected, which results in an absolute error of about 4 pixels (cut off at 1.5 pixels in the figure). For somewhat less extreme noise levels, the average absolute error is about 0.1 pixels. To demonstrate the noise levels, the image shown in figure 4.6 is distorted by gain and offset nonuniformity with standard deviation 0.3 and 25 respectively. 


\subsection{Comments on implementation}

When implementing this motion estimation algorithm, the problem arises about which two images to relate in the estimation process.

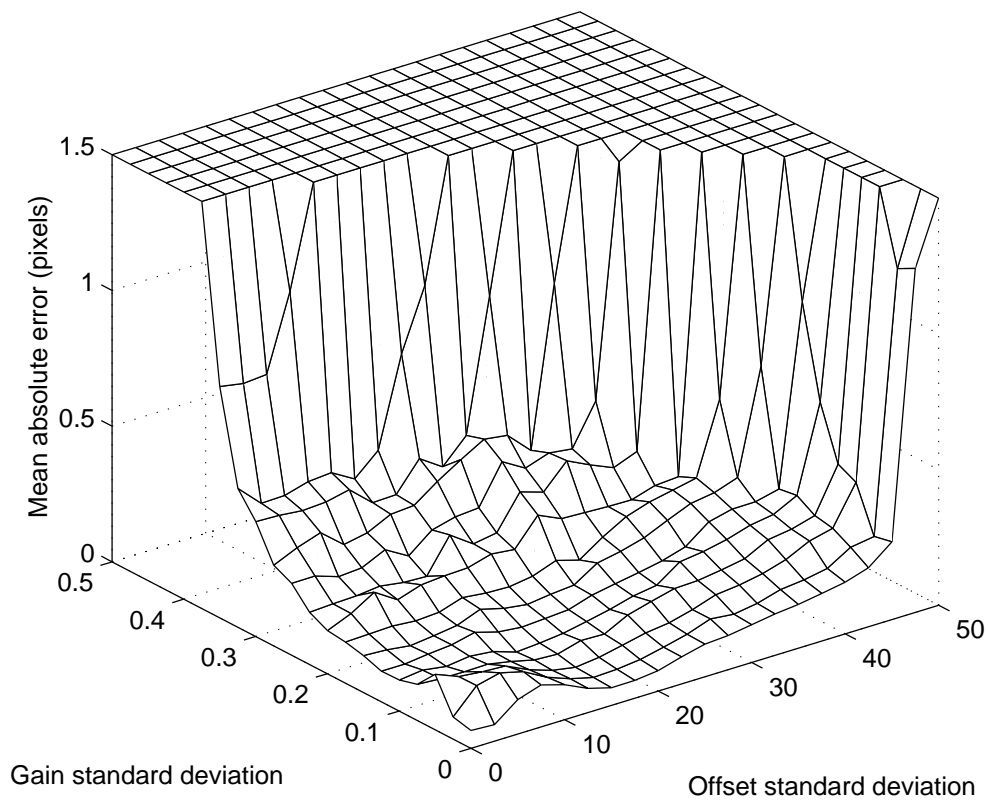

Figure 4.5: Mean absolute error of translation estimates for various levels of gain and offset nonuniformity in an 8-bit grayscale image.

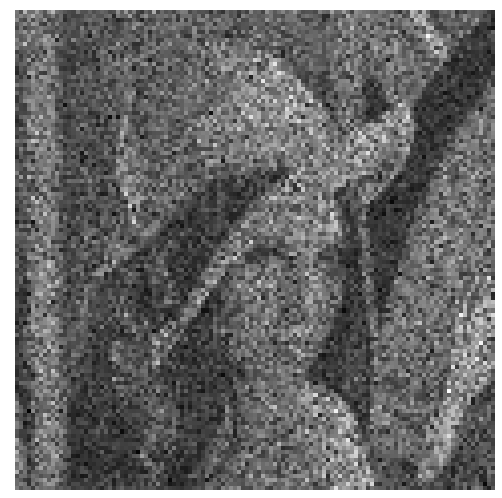

Figure 4.6: An 8-bit grayscale image with normal distributed gain and offset nonuniformity, standard deviations 0.3 and 25 respectively. 
Unless the sensor is restricted to move only a short distance from the initial position, it will soon be impossible to relate new frames to the first frames since they obviously share less and less of the scene data.

A reasonable solution would be to relate each new frame to the previous frame. This may however prove undesirable. There is always a small error in each individual motion estimate, hopefully no larger than about 0.1 pixels. When constantly using the previous frame as a reference for the new estimate, this small error is accumulated and may well grow to several pixels - resulting in completely useless motion estimates.

To avoid accumulating errors, the reference frame should be kept the same for as long as possible. This can be done by changing reference frame only when the distance to the new frame is approaching the limit of the estimation algorithm.

There are problems with this method as well, though. Chances are that an image frame with unusually large estimation error is chosen as the reference frame. In that case, that error will be present in all the following frames. A possible solution could be to examine the reference frame candidate more closely before actually selecting it. For example, a frame with an absolute translation vector that deviates rather much from the translation vectors of following and preceding frames would not be an ideal choice as a new reference frame. 



\section{Chapter 5}

\section{Registration-based nonuniformity correction}

\subsection{Motion compensating methods}

With motion estimation, each image in the sequence can be spatially related to previous images. This is an advantage for further image processing. For example, the input images can be translated accordingly and merged together to form a new, larger image. Figure 5.1 shows such a panoramic image created from one of the MSM image sequences. The creation of a panoramic image is the first step in a registration-based nonuniformity correction method known as motion compensated average [8].

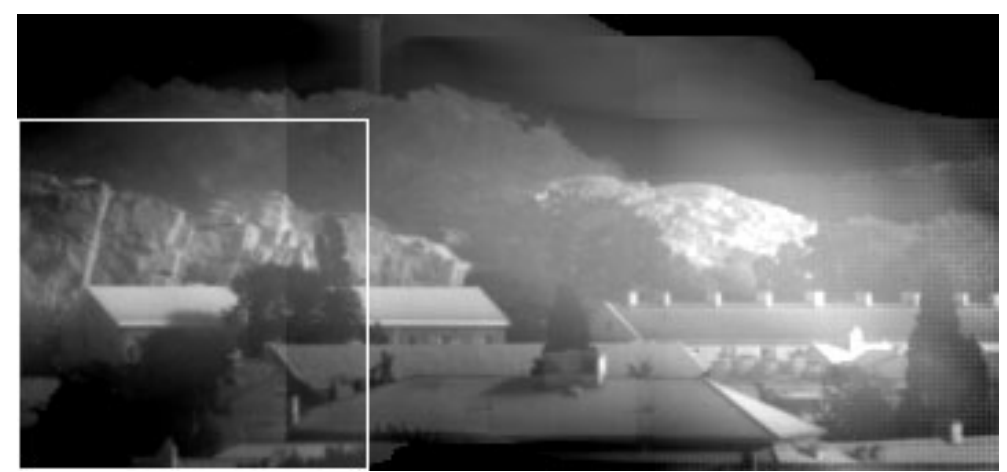

Figure 5.1: Panoramic image. The last inserted frame is indicated.

The word average indicates that the panoramic image is created 
by averaging image frames together. This means that the values of all pixels that represent the same position in the scene should contribute equally to the resulting value in the panoramic image. In practice, a counter is kept for each position in the panoramic image, indicating the number of pixels used so far to create the value for this position. Each new image that is to be added is first corrected using the latest correction parameters. It is then translated and sub-pixel interpolated to the right position and merged with the panoramic image using a pixel-wise variant of the recursive averaging formula (3.3).

Actually, this averaging into a panoramic image is in itself a nonuniformity correction method. Assuming that motion estimation has been successful, the scene data in the new image is the same as at the corresponding position in the panoramic image. The fixed pattern noise however, is not. Thus, for each image added to the panoramic image, the true scene is enhanced while the fixed pattern noise is reduced. Because of this, the panoramic image is considered to be an estimate of the true scene. Returning the corresponding part of the panoramic image is thus a simple nonuniformity correction. Extracting the actual correction parameters is however often useful. For example, parameter sets from several frames can be averaged together to form an improved parameter estimate. Knowing the parameters also allows for correction of all subsequent frames with minimal computation and without registration.

For the offset-only model (3.2), the offset parameters are given by simply subtracting the observed image from the true estimate. For the linear model (3.1), gain and offset parameters can be found, for example, by using a least-square fit. This of course requires more than one true-observed image pair to give any useful results.

One estimate of the correction parameters is now delivered by the algorithm for each input image. By combining the estimates from several frames, the correction parameters would intuitively improve. For example, temporal averaging using the recursive IIR filter (3.3) can be used to make all the given parameter estimates contribute equally to the final parameters. A more sophisticated model could be used if the temporal behavior of the parameters is known, in which case a newly estimated value might be weighted differently than a value estimated some time ago.

Naturally, this algorithm assumes a mostly static scene. If the scene does not remain sufficiently unchanged over time, the generated 
panoramic image is not very useful. The static scene assumption is common for registration-based algorithms.

\subsection{Crossing path}

So far, knowledge of the image motion in a sequence has been used to construct a panoramic image for further nonuniformity processing.

The image motion can also be used to study the difference in detector response in a more algebraical sense. For example, if the relative translation between two observed images is exactly $(1,0)$ pixels, two detector elements with the relative distance $(1,0)$ units have observed the very same scene irradiance and should ideally produce the same output value. Of course, this reasoning assumes a static scene, as mentioned earlier. Any deviation is caused by detector nonuniformities. Translation vectors ending up at exactly integer pixel units is however rather improbable.

It has been shown by Ratliff et al. [7] that for pure horizontal or vertical sub-pixel translations, the responses of two detectors at unity distance can be accurately related through extrapolation. An elegant nonuniformity correction method for offset-only calibration (3.2) can be derived that unifies the offset terms to one unknown but global value. Another approach to this problem is to specifically move the image sensor according to a predetermined pattern. This is suggested in the patent of O'Neil, described in [2], along with a method for both gain and offset calibration (3.1).

The algorithm introduced here is called crossing path and is based on these two methods. Both gain and offset correction parameters are estimated, and the constraint of pure horizontal or vertical motion is somewhat relaxed.

\section{Analyzing detector movements}

The main idea of this algorithm is to find all pairs of images that are related by translation vectors with integer vertical and horizontal components. When such a pair has been found, it is possible to identify different detectors that observe the exact same scene value.

For each new input image, motion estimation delivers the corresponding position of the image origin relative to a previous image. This means that the distance to every previous position can easily be calculated. If any of these distances are sufficiently close to an 
integer value, a suitable image pair has been found. In figure 5.2, a detector path of motion is shown where the registered images 5 and 10 are quite close to being separated by $(1,1)$ pixels. However, it is highly improbable that the distance is exactly integer valued.

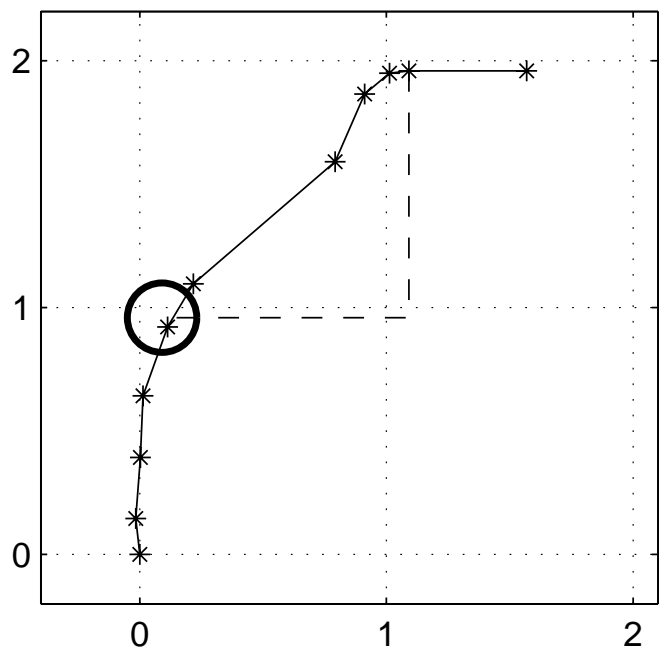

Figure 5.2: A detector motion path. The distance between image 5 and 10 is almost $(1,1)$ pixels.

The probability of integer pixel distances can be increased by interpolating the image sequence to create in-between images. In practice, this means that the number of image positions is increased, allowing for an improved distance match. This procedure can be repeated infinitely. Eventually, it is no longer discrete image positions that are compared, but continuous segments of the detector motion path.

Finding integer valued pixel distances can be expressed as finding crossing points between the new path segment and all the previous segments, duplicated horizontally and vertically in unity steps, representing the motion paths for all elements in the detector array. This search for intersections is what gives the algorithm its name. Each crossing check can be performed quickly since the segments can be expressed as linear equations with boundaries. Figure 5.3(a) shows the detector motion path from figure 5.2 with duplicates at $(0,1)$ and $(1,1)$ pixels, representing three individual elements in the detector array. The two paths separated by $(0,1)$ pixels gets rather close at 
the first and fifth image respectively, indicating that the first and the fifth image recorded are related almost exactly by a translation of one pixel vertically. Nevertheless, this is disregarded, since the paths do not cross each other. After another couple of frames, however, the paths separated by $(1,1)$ pixels do. The crossing point is shown in more detail in figure 5.3(b), indicated by a circle. At this point, two images are related by a translation of exactly $(1,1)$ pixels. Unfortunately, no images have been recorded at this specific point in time, but estimates can be obtained by interpolation. In this case, images 5 and 6 are interpolated into one image and images 10 and 11 are interpolated into another.

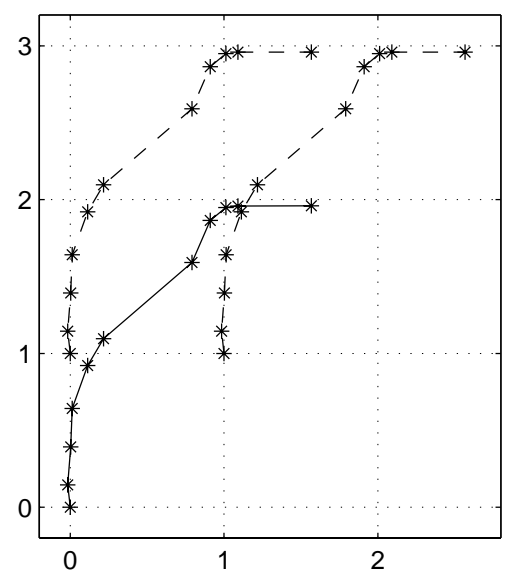

(a) Three motion paths

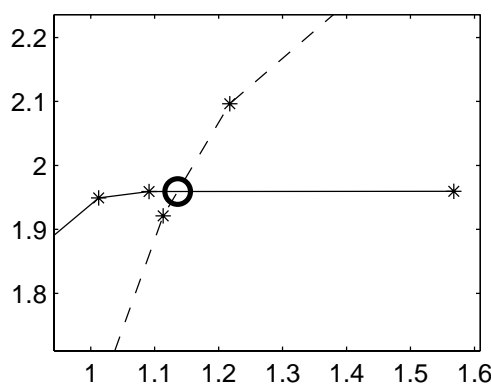

(b) Close up of crossing point

Figure 5.3: Three detector motion paths for the first 11 images.

If the length of the segment is greater than one, i.e. the relative translation between the two images is greater than one pixel, the interpolation might introduce too large errors and the crossing should be disregarded. The crossing should also be disregarded if too much time has passed between the two observations, since the assumption of a static scene might not be valid.

Checking a new path segment for crossings with all previous segments at all possible detector locations is a time consuming process that can be simplified. Consider a square with the size of one pixel. The center of the square represents a reference origin, from which 
a motion path is constructed. The motion path extends with one segment for each input image, inevitably crossing the square border at some time. When this happens, the corresponding segment is divided into two parts, one that reaches the border and another that continues from the opposite side of the square. Thus, the motion path is trapped inside the square, wrapping around cyclicly as it tries to cross a border. Every time the path is wrapped around, a distance vector is updated that indicates the integer horizontal and vertical difference to the actual position of the path. Every new segment is tagged with its corresponding distance vector. The resulting motion path is then checked for crossings with itself. When a crossing is detected, the detector element has reached a position already visited before, but with an integer horizontal and vertical relative translation, indicated by the difference in the distance vectors of the two crossing segments. It is thus sufficient to perform crossing detection only on one motion path, as the distance vectors indicate which two original motion paths that have actually crossed each other. The motion path from figure 5.2 is built up in this manner in figure 5.4. Eventually a crossing point is detected for two segments, one created when the distance vector was $(0,1)$ and the other when the distance vector was $(1,2)$. The difference is $(1,1)$, which indicates that the images are related by a translation of $(1,1)$ pixels, just as before.

For each detected crossing point, the algorithm delivers an image pair and a translation vector that indicates the integer horizontal and vertical translation between the images.

\section{Correcting offset nonuniformity}

The first step in this correction algorithm is to consider only offset nonuniformity, which means that the gain parameters are assumed to be unity.

With this assumption, two pixels $x_{a}$ and $x_{b}$ in a delivered image pair as described above, with relative distance given by the corresponding translation vector, can be written as $x_{a}=z+o_{a}$ and $x_{b}=z+o_{b}$. The two pixels represent the same scene value $z$, observed by two detectors with additive fixed pattern noise $o_{a}$ and $o_{b}$. Subtracting these pixel values effectively cancels out the scene value, leaving only

$$
x_{a}-x_{b}=z+o_{a}-z-o_{b}=o_{a}-o_{b}
$$




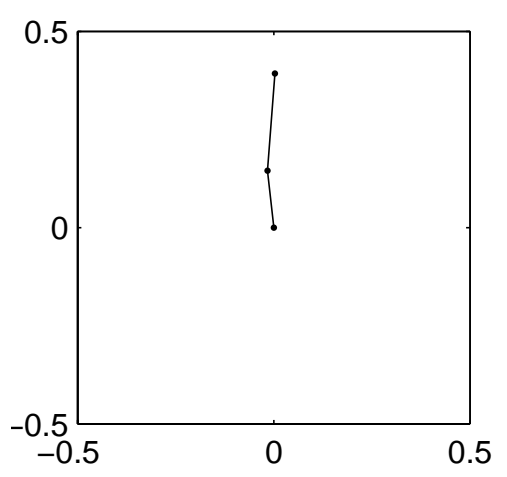

(a) Distance vector: $(0,0)$

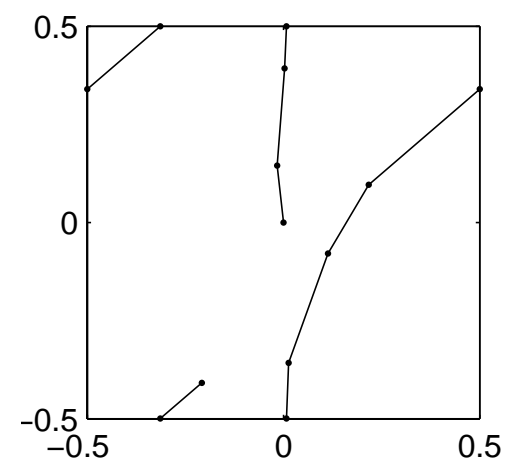

(c) Distance vector: $(1,2)$

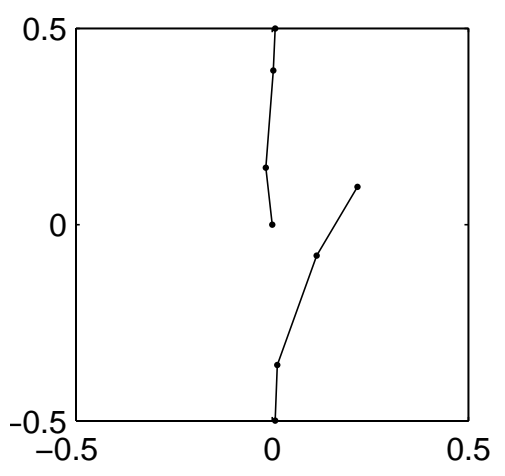

(b) Distance vector: $(0,1)$

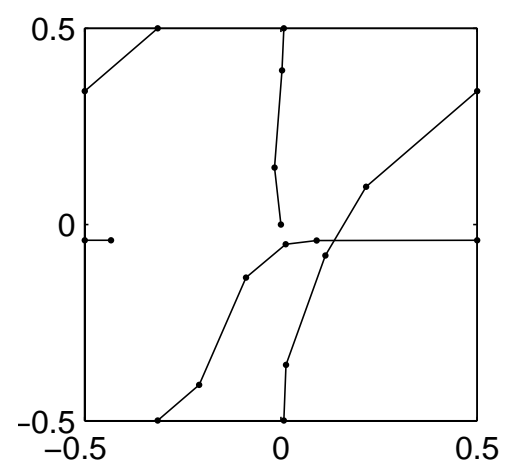

(d) Distance vector: $(1,3)$

Figure 5.4: A motion path constrained within a pixel-sized square.

Adding this value to the pixel $x_{b}$ has the effect of changing the corresponding offset nonuniformity to that of pixel $x_{a}$.

Of course, pixels $x_{a}$ and $x_{b}$ are not the only two pixels that are related like this. Pixel $x_{b}$ can in turn be related to a third pixel, $x_{c}$. Using the same method, the offset nonuniformity of $x_{c}$ can be changed to that of pixel $x_{b}$, which again can be changed to that of pixel $x_{a}$ using (5.1). This effect accumulates along the entire image. The whole procedure is shown in the following figures.

Figure 5.5 shows a pair of images related by a vertical shift of two pixels. Subtraction of pixels representing the same scene value 


\begin{tabular}{|l|l|l|}
\hline$Z_{11}+O_{11}$ & $Z_{12}+O_{12}$ & $Z_{13}+O_{13}$ \\
\hline$Z_{21}+O_{21}$ & $Z_{22}+O_{22}$ & $Z_{23}+O_{23}$ \\
\hline$Z_{31}+O_{31}$ & $Z_{32}+O_{32}$ & $Z_{33}+O_{33}$ \\
\hline$Z_{41}+O_{41}$ & $Z_{42}+O_{42}$ & $Z_{43}+O_{43}$ \\
\hline$Z_{51}+O_{51}$ & $Z_{52}+O_{52}$ & $Z_{53}+O_{53}$ \\
\hline$Z_{61}+O_{61}$ & $Z_{62}+O_{62}$ & $Z_{63}+O_{63}$ \\
\hline$Z_{71}+O_{71}$ & $Z_{72}+O_{72}$ & $Z_{73}+O_{73}$ \\
\hline
\end{tabular}

(a)

\begin{tabular}{|l|l|l|}
\hline$Z_{31}+O_{11}$ & $Z_{32}+O_{12}$ & $Z_{33}+O_{13}$ \\
\hline$Z_{41}+O_{21}$ & $Z_{42}+O_{22}$ & $Z_{43}+O_{23}$ \\
\hline$Z_{51}+O_{31}$ & $Z_{52}+O_{32}$ & $Z_{53}+O_{33}$ \\
\hline$Z_{61}+O_{41}$ & $Z_{62}+O_{42}$ & $Z_{63}+O_{43}$ \\
\hline$Z_{71}+O_{51}$ & $Z_{72}+O_{52}$ & $Z_{73}+O_{53}$ \\
\hline$Z_{81}+O_{61}$ & $Z_{82}+O_{62}$ & $Z_{83}+O_{63}$ \\
\hline$Z_{91}+O_{71}$ & $Z_{92}+O_{72}$ & $Z_{93}+O_{73}$ \\
\hline
\end{tabular}

(b)

Figure 5.5: An image pair with translation vector $(0,2) . Z$ represents a true value that has been distorted by an offset term $O$.

$z_{y x}$ yields the image in figure 5.6(a). No values are calculated for the first two rows since that would require data from outside the first image frame. As expected, each offset term is present twice, with same relative spacing as the relative shift between the images. This is exploited by performing a cumulative sum over all elements separated by this relative spacing. In this case, the cumulative sum is calculated for every second pixel value in each column. The resulting image is shown in figure 5.6(b). This image contains offset term

\begin{tabular}{|c|c|c|}
\hline 0 & 0 & 0 \\
\hline 0 & 0 & 0 \\
\hline$O_{11}-O_{31}$ & $O_{12}-O_{32}$ & $O_{13}-O_{33}$ \\
\hline$O_{21}-O_{41}$ & $O_{22}-O_{42}$ & $O_{23}-O_{43}$ \\
\hline$O_{31}-O_{51}$ & $O_{32}-O_{52}$ & $O_{33}-O_{53}$ \\
\hline$O_{41}-O_{61}$ & $O_{42}-O_{62}$ & $O_{43}-O_{63}$ \\
\hline$O_{51}-O_{71}$ & $O_{52}-O_{72}$ & $O_{53}-O_{73}$ \\
\hline
\end{tabular}

(a) Result after subtraction

\begin{tabular}{|c|c|c|}
\hline 0 & 0 & 0 \\
\hline 0 & 0 & 0 \\
\hline$O_{11}-O_{31}$ & $O_{12}-O_{32}$ & $O_{13}-O_{33}$ \\
\hline$O_{21}-O_{41}$ & $O_{22}-O_{42}$ & $O_{23}-O_{43}$ \\
\hline$O_{11}-O_{51}$ & $O_{12}-O_{52}$ & $O_{13}-O_{53}$ \\
\hline$O_{21}-O_{61}$ & $O_{22}-O_{62}$ & $O_{23}-O_{63}$ \\
\hline$O_{11}-O_{71}$ & $O_{12}-O_{72}$ & $O_{13}-O_{73}$ \\
\hline
\end{tabular}

(b) Result after cumulative sum

Figure 5.6: Subimages generated by the algorithm.

differences distributed in such a way that when added to an image from the sequence, the existing offset terms are replaced with one 
of two offset terms, belonging to the two first detector elements in each column. This means that the number of different offset terms has been reduced from $N^{2}$ to $2 N$, a partial nonuniformity correction. The corrected version of the image in figure 5.5(a) is shown in figure 5.7 .

\begin{tabular}{|l|l|l|}
\hline$Z_{11}+O_{11}$ & $Z_{12}+O_{12}$ & $Z_{13}+O_{13}$ \\
\hline$Z_{21}+O_{21}$ & $Z_{22}+O_{22}$ & $Z_{23}+O_{23}$ \\
\hline$Z_{31}+O_{11}$ & $Z_{32}+O_{12}$ & $Z_{33}+O_{13}$ \\
\hline$Z_{41}+O_{21}$ & $Z_{42}+O_{22}$ & $Z_{43}+O_{23}$ \\
\hline$Z_{51}+O_{11}$ & $Z_{52}+O_{12}$ & $Z_{53}+O_{13}$ \\
\hline$Z_{61}+O_{21}$ & $Z_{62}+O_{22}$ & $Z_{63}+O_{23}$ \\
\hline$Z_{71}+O_{11}$ & $Z_{72}+O_{12}$ & $Z_{73}+O_{13}$ \\
\hline
\end{tabular}

Figure 5.7: Cumulative sum added to original image

This correction procedure is applied to all the following images in the sequence. Furthermore, whenever new image pairs are delivered, hopefully with translation vectors in other directions, the number of offset parameters is even more reduced. Eventually, the entire image shares an unknown but common offset parameter.

\section{Correcting gain nonuniformity}

The gradients of the outputs of two detector elements following the same path between two specific scene points should be equal. Any deviation is caused by variations in the gain parameters of those elements. With this knowledge it is possible to incorporate gain parameter estimation in the algorithm.

The reason for involving a gradient is to eliminate the offset nonuniformity. The ratio between the two detector gradients then gives an estimate of the gain parameter. As an example, consider two detector elements $a$ and $b$ that have observed the same scene position on three occasions:

$$
\begin{array}{ll}
z_{1} \cdot g_{a}+o_{a} & z_{1} \cdot g_{b}+o_{b} \\
z_{2} \cdot g_{a}+o_{a} & z_{2} \cdot g_{b}+o_{b} \\
z_{3} \cdot g_{a}+o_{a} & z_{3} \cdot g_{b}+o_{b}
\end{array}
$$


The offset terms are eliminated by constructing gradients:

$$
\begin{array}{ll}
\left(z_{1}-z_{2}\right) \cdot g_{a} & \left(z_{1}-z_{2}\right) \cdot g_{b} \\
\left(z_{2}-z_{3}\right) \cdot g_{a} & \left(z_{2}-z_{3}\right) \cdot g_{b}
\end{array}
$$

The scene data can be eliminated by dividing the gradients, resulting in two estimates of $g_{a} / g_{b}$. By multiplying the output of detector $b$ with this estimate, the corresponding gain parameter can be replaced with that of detector $a$. Naturally, since all elements in the array are spaced equally, similar relations can be found between all the other detectors in the array, allowing for a gain parameter reduction just like the offset term reduction described earlier, this time using a cumulative product instead of a sum.

\section{Comments on the implementation}

As described, the accumulation of correction terms is performed over an entire image. Unfortunately, this means that errors in one correction term may be distributed to several other terms. To overcome this, the accumulation is allowed only for a limited number of terms, and is then reset. This can for example mean that pixels 1-20 share one offset term, pixels 21-40 share another, and so on. Errors in one term is then only distributed to the other pixels in that group. Figure 5.8 shows the difference. Disturbance at the top gives rise to errors that accumulate down over the entire image (a). When using a maximum accumulation length of 20 pixels, the errors are only spread a short distance (b).

As previously described, the accumulation is performed so that all pixels share the offset term of the first pixel. It is of course possible to instead share the offset term of the last pixel, by changing the direction of the cumulative sum. In the implementation, this direction is swapped for each new frame to cancel out any border effects. 


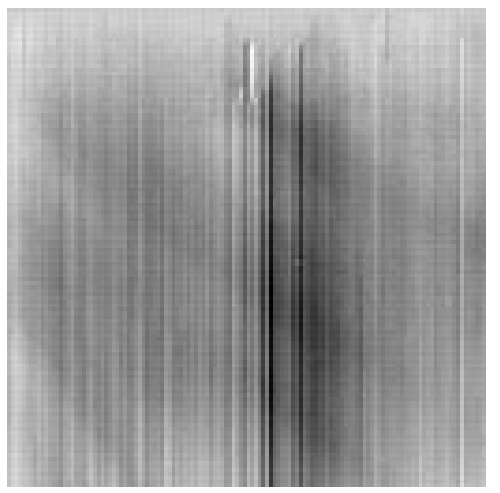

(a) No accumulation limit

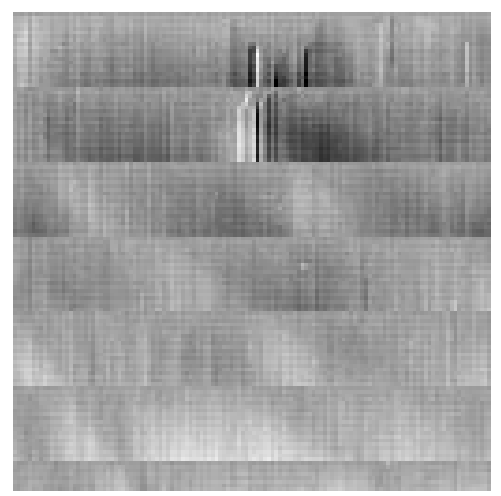

(b) 20 pixel limit

Figure 5.8: Error accumulation in crossing path algorithm. 



\section{Chapter 6}

\section{Evaluation}

\subsection{Test sequences}

Eight different sequences of 400 frames each have been recorded in Göteborg using the MSM system. These sequences were extensively used for testing and tuning the algorithms during the implementation stage. Another four MSM sequences were recorded some months later for evaluation purposes, along with a couple of sequences from the more sophisticated QWIP camera and the one-dimensional sensor of the IRST system. Synthetic sequences have also been constructed for some specific evaluation.

\subsection{Evaluation methods}

The nonuniformity correction performed on the sequences is not designed for any specific field of application other than to produce high quality images. This complicates the evaluation, since the human visual system and an artificial image processing system usually have different opinions on what constitutes a high quality image. The evaluation is carried out with this in consideration, using both calculated quality measures and opinions from human observers.

\subsection{Temporal noise}

When evaluating the algorithms, it is useful to know the magnitude of the temporal noise that always is present in the sequences, since this sets a limit for image quality in the resulting sequences. 
This magnitude can be measured by studying the output from a single detector element in a sequence without image motion. Unfortunately, none of the available sequences are completely motionless. A reasonable estimate should be possible to obtain anyway by ignoring the low frequency signal variations in a slowly varying image sequence. The noise level can then be estimated visually from a signal plot. Removing low-frequency information by highpass filtering may simplify this procedure even more. Figure 6.1 shows the output from a single detector element in one of the MSM sequences, suggesting that the temporal noise magnitude usually is below 10 units, with a standard deviation of 3-4 units.

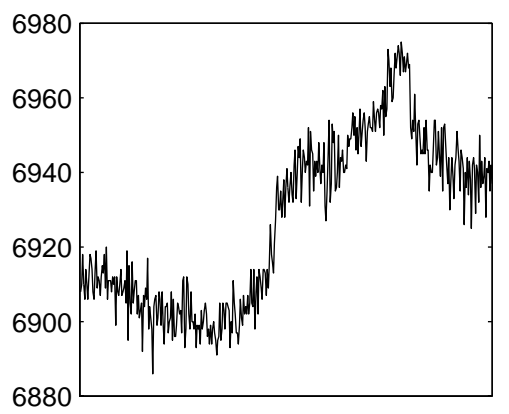

(a) Original detector output

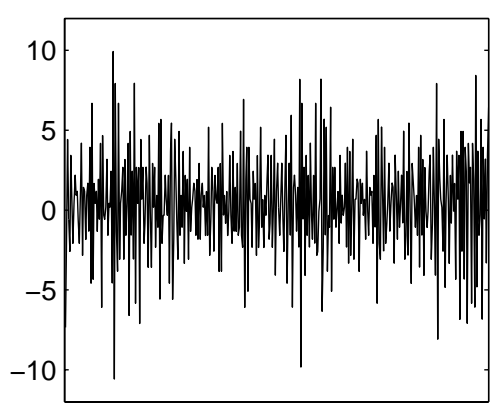

(b) Highpass filtered

Figure 6.1: The output from a single MSM detector element.

\subsection{One-image correction}

The one-image correction method described in section 3.4 is able to improve image quality in the test sequences. Being a static preprocessing correction procedure, this is mainly a way of reducing the time of convergence for the described nonuniformity correction methods. However, the differences between the methods also become harder to demonstrate, especially in print. For the following evaluation, this one-image correction will therefore not be performed unless otherwise stated. 


\subsection{Temporal highpass filter}

The temporal highpass method has the advantage of being very fast and easy to implement. It is however an offset-only correction algorithm, capably only of reducing additive noise.

The fixed pattern noise estimate that is subtracted from the image frames is generated by a temporal mean of the sequence. This gives appropriate results only when all detector elements have been exposed to roughly the same range of scene irradiance. If, for example, some elements only observe bright parts of the scene, their corresponding offset correction term will be too high. This means that until a sufficient number of frames have been processed, bright scene areas give rise to burned-in inverse patterns in the output sequence, much like the ghosting artifacts described in section 3.3.

The number of frames needed depends on the scene and the motion. Most tested sequences require several hundred frames to reduce the ghosting effects to an acceptable level. Usually, some traces were still visible at the end of the sequences. The fixed pattern noise was however rather quickly suppressed with good results.

Note that since the temporal mean of the sequence is constantly subtracted, the range of intensity values in the output sequence is centered around zero.

\subsection{Constant statistics}

The constant statistics algorithm is basicly an extension of the temporal highpass filter to include gain parameter estimation. Therefore it is not very surprising that the two methods produce very similar results. The pattern burn-in artifacts described above are visible here as well, actually even more than before since the effect now is present in both the offset and the gain parameter images. As for the convergence rate, the image quality at the end of the sequence is roughly the same as for the temporal highpass method. However, the typical resulting gain parameter image, shown in figure 6.2 , is not similar at all to the expected gain variation from figure 3.1. Some patterns in the image are actually traces of the observed scene, which indicates that this is not a very good gain estimate. This algorithm probably needs a lot more image frames to converge than the 400 frames available in the test sequences — about 10000 frames are suggested in [4]. 


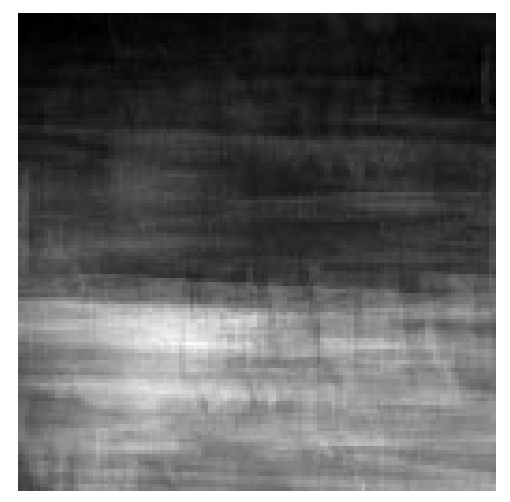

Figure 6.2: Typical resulting gain parameters for constant statistics algorithm.

This algorithm assumes that the true mean value of the scene is 0 and the mean deviation is 1 . Because of this, the intensity values of the resulting sequences are scaled to fit the assumptions. In order to objectively compare results from different correction methods, it may be better to leave the range of intensity values approximately unchanged. This can be done by modifying the assumptions so that the mean gain correction becomes unity.

\subsection{Motion compensated average}

For the registration-based methods, motion estimation is an essential part of the algorithms. If motion estimation fails, or the assumption of a static scene is violated too much, severe distortions may be introduced in the resulting sequences.

Since this method is based on performing a running average of motion compensated incoming frames, it is vulnerable to ghosting. Most of these artifacts can however be successfully suppressed by using the simple deghosting principle described in section 3.3. Actually, most of the test sequences show no signs of introduced artifacts.

The offset-only version of this method is able to reduce fixed pattern noise very quickly, especially high-frequency spatial noise. Some low-frequency shading effects remain visible, probably multiplicative in origin. For a human observer, such noise is however less disturbing than high-frequency noise. 
Estimation of the gain parameters involves a least-square fit of observed frames to estimated true frames. Achieving good results with this procedure has however proven to be difficult. For example, figure 6.3 shows a plot of the observed scene values for a specific detector element along with the corresponding estimated true values from the panoramic image. Finding a linear relation between the two plots is quite problematic. The gray areas indicate extreme situations where one plot is approximately constant while the other is varying heavily. Trying to find a least-square fit for these plots may well result in zero or negative gain parameters, which in turn forces the offset parameter to levels equal to or greater than the total irradiance value.

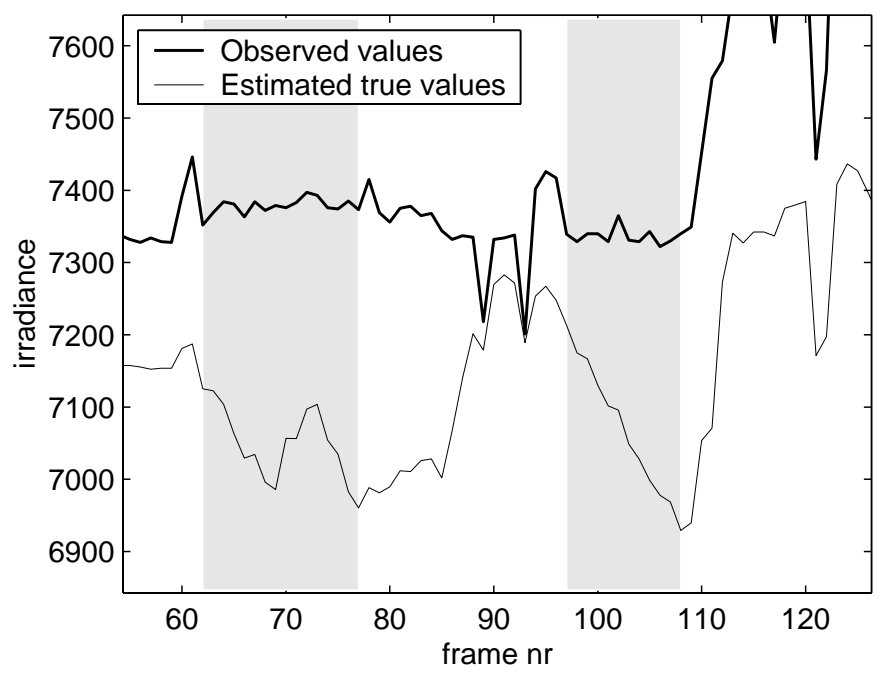

Figure 6.3: Observed and estimated values seem uncorrelated, especially within the gray areas.

This type of problem occurs when fixed pattern noise causes shading artifacts in the panoramic image. For example, signal intensity usually decreases at the edges of the source images. Because of this, a part of the scene that has been observed only at the image edges contributes to the panoramic image with too low intensity. This is unfortunate, since the panoramic image is used as an estimate of the true scene. Illustrated in figure 6.4, the smiley face is observed for a long time in a shaded region (figure a). Because of this, the true 
estimate is assumed to be very dark. When finally moving the sensor towards the smiley (figure b), a detector element at the center of the sensor will observe a constant brightness, while the estimated true value decreases. The observed signal and the estimated true signal are uncorrelated, which resembles the situation in figure 6.3.

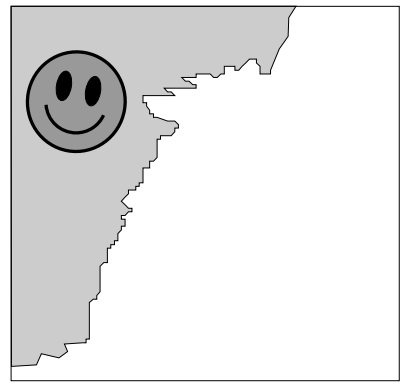

(a)

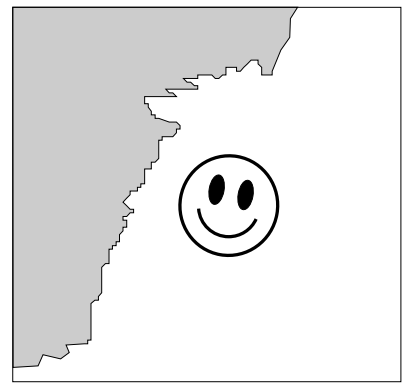

(b)

Figure 6.4: Fixed pattern noise disturbing true scene estimate.

The probability of uncorrelated signals can be reduced by fine tuning the deghosting algorithm and performing one-image correction that removes most of the low-frequency noise. The effects can however not be completely removed.

Another problem is illustrated in figure 6.5. At a first glance, a linear relation between the two plots seems easy to find - the magnitude relation is about 2:1 and the two plots are offset by some hundred units. However, if the observed values are divided by 2 , the offset term needs to be about 3500 units to reach the level of the estimated true values. This may of course be the correct transformation, but it is also possible that the estimated true values have been distorted somehow. For example, both inserting and fetching a value from the panoramic image involves bilinear interpolation, which has a low-pass filtering effect. If this is what causes the factor 2 in magnitude change, the resulting correction parameters are far from correct.

For the test sequences, the fixed pattern noise is actually reduced in some cases. However, the algorithm often introduce disturbing artifacts which suggests that the correction is indeed scene dependent.

Solutions to these problems are hard to find. For starters, a lot of 


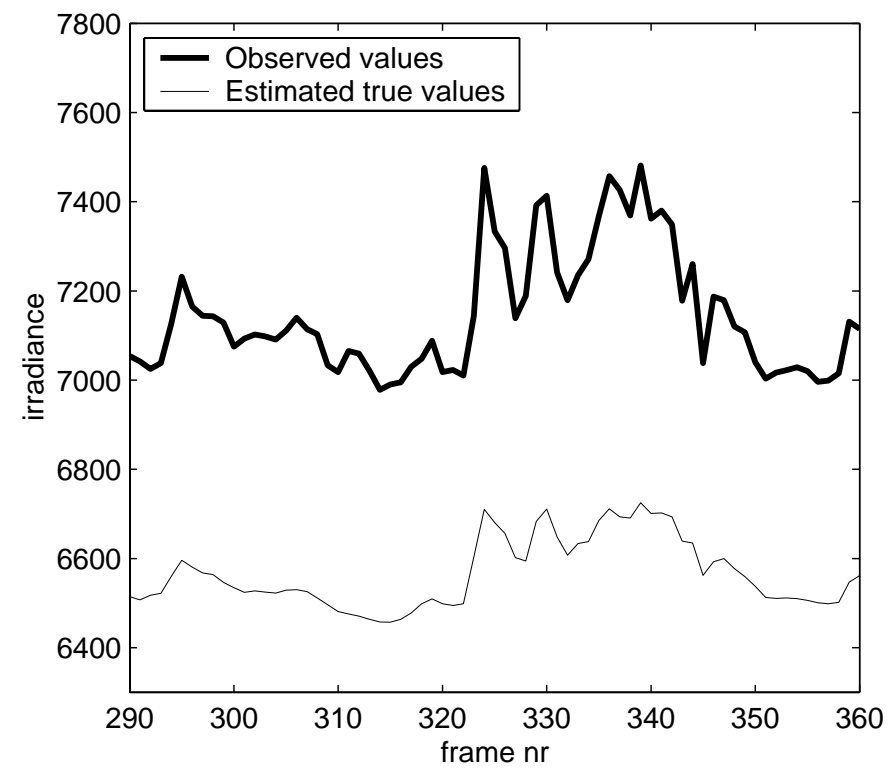

Figure 6.5: Observed and estimated value for a detector element.

procedures for detecting valid input data have to be included in the algorithm, and the parameters must be updated very slowly. Still, the offset-only version always gives better results. A possible conclusion to be drawn is that the true scene estimate is good enough only for additive noise correction. The least-square method is considered too unstable and will not be evaluated further.

A way of improving the offset-only correction is to introduce a simple algorithm that reduces low-frequency noise and shadows. Such an algorithm is already available - the homomorph filter of the oneimage correction procedure (section 3.4). Repeating this one-image correction for each input frame and averaging the resulting gain correction images over time results in a method that at least improves the offset-only correction to some degree.

\subsection{Crossing path}

Theoretically, this is the fastest nonuniformity correction method, ideally requiring only two frames to remove all additive fixed pattern 
noise. In practice, the image sequences contain temporal noise, dead pixels and moving scene objects that disturb the algorithm. Nevertheless, some of the test sequences are completely cleaned from all visible fixed pattern noise within only 50 frames.

The in-between image interpolation is assumed to be valid only if the two source images are related by a translation of one pixel or less. This is a drawback of the crossing path algorithm. For sequences where the image-to-image translation is always more than a pixel, no correction at all is performed. If such problems arise, it may be necessary to modify the algorithm to find detector element overlaps in some other way, for example by checking for detector translations close to integer distances.

The crossing path algorithm is especially sensitive to errors in motion estimation and violations of the static scene assumption. Examples of such artifacts are showed in figure 6.6(a), where a truck drives through the lower right part of the scene, and in figure 6.6(b), where a very hot spot in the lower part of the scene causes noise since it is so small that it sometimes disappears due to limited resolution. Most artifacts that are introduced by the algorithm are however cancelled out rather quickly, since the algorithm itself is adapting very fast to new fixed pattern noise.

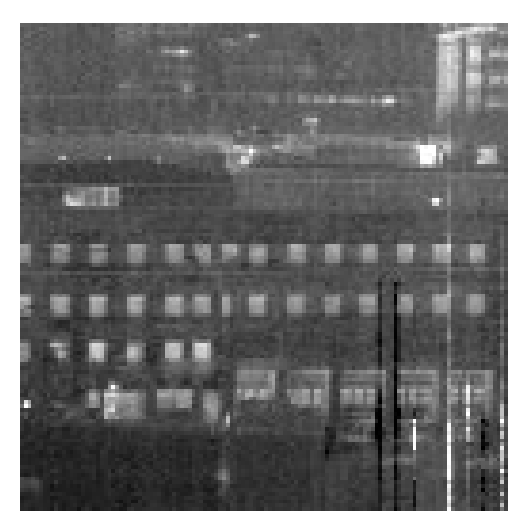

(a) Moving objects in scene

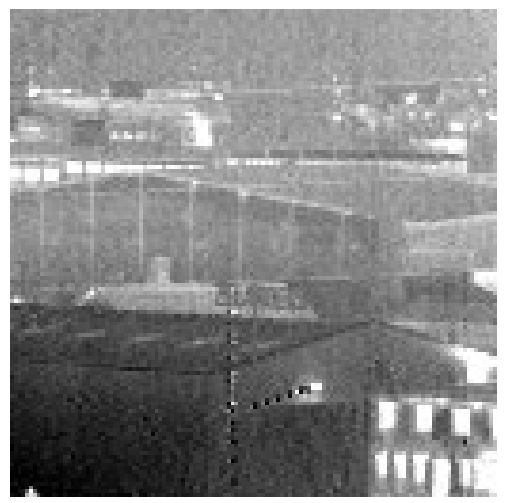

(b) Tiny hot spot disappears

Figure 6.6: Artifacts due to violated static scene assumption.

Unfortunately, the gain parameter estimation does not work as good as expected. As described in section 5.2, the offset terms are 
first removed by constructing gradients of each detector output. Relations between gain parameters are then calculated by dividing the gradients. The results are however not easily interpreted. As an example, consider the situation in figure 6.7, where two detector elements have observed the same point of the scene on six occasions.

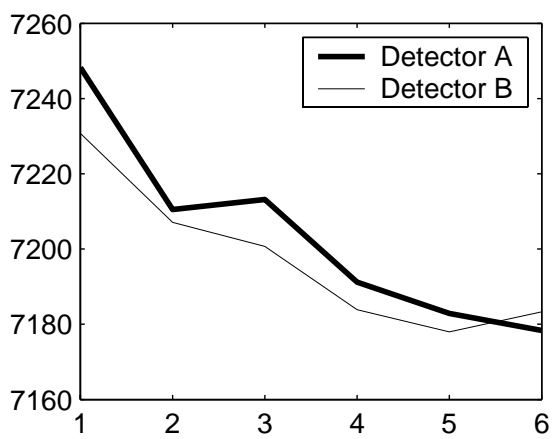

(a) Detector outputs

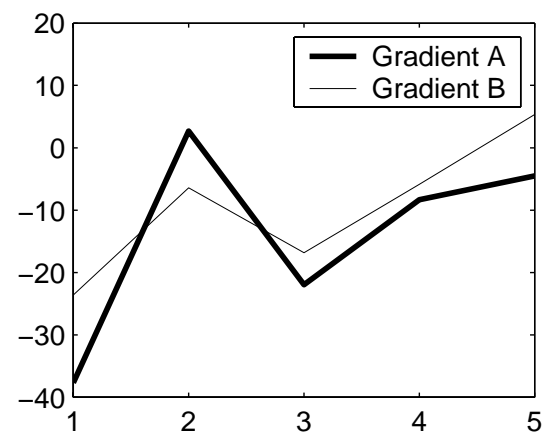

(b) Gradients

Figure 6.7: Signals from two detectors observing the same scene points on six occasions.

Five estimates of the gain parameter relation $g_{A} / g_{B}$ can be computed by dividing each pair of gradient values. For this particular case, the estimates are:

$$
\begin{array}{lllll}
1.60 & -0.41 & 1.31 & 1.42 & -0.84
\end{array}
$$

As can be seen, the uncertainty is so high that it is practically impossible to use this method for gain parameter estimation. Inaccuracy in translation estimation, in-between image interpolation and changes in the scene all contribute to distortions, but even in the most ideal case, temporal noise alone can be enough to cause such variations in the signal outputs that the gradient ratios become unreliable or even negative. Also, when two observed values are nearly identical, the algorithm includes a division with a value close to zero, introducing large numerical errors.

The method can be modified to counter some of these problems. Instead of assuming unity gain parameters for offset-only correction, the offset terms can be assumed to be zero for gain-only correction. 
Gain correction can then be performed exactly like the offset correction by changing the subtractions to divisions. The revised algorithm now works in two steps:

- Assume that fixed pattern noise is completely additive.

- Perform offset-only correction.

- Assume that fixed pattern noise is completely multiplicative.

- Perform gain-only correction.

Hopefully, the correction procedure will converge so that multiplicative noise is eventually removed by the gain parameters and additive noise by the offset parameters. If this really is the case is hard to say. For most test sequences, the resulting gain and offset correction images end up very similar. There is also no visible improvement of image quality compared to the offset-only correction. 


\section{Chapter 7}

\section{Results}

\subsection{Computational speed}

Optimizing algorithms for speed is not a specific goal for this thesis. Nevertheless, it might be interesting to see how fast the different methods are. Table 7.1 shows a rough average of how many frames per second the algorithms are able to process. The tests have been performed in MATLAB on a dual Pentium 3 system using sequences of 400 frames, each $128 \times 128$ pixels.

\begin{tabular}{lc} 
Method & Frame rate \\
\hline Temporal highpass filter & 36.6 \\
Constant statistics & 24.2 \\
Motion compensated average & 4.8 \\
Crossing path, offset only & 5.3 \\
Crossing path, offset and gain & 4.3
\end{tabular}

Table 7.1: Average frame rate for the NUC methods.

As expected, the registration-based methods require much more processing time than the simpler ones, partly due to the need for motion estimation. It should be noted that the computation time for the crossing path method depends on how many valid motion path collisions that are detected. For example, the parameters were updated 280 times in one of the sequences and 1070 times in another. 


\subsection{Reference images}

Images of a reference surface with uniform temperature, one at $0^{\circ} \mathrm{C}$ and another at $30^{\circ} \mathrm{C}$, have been recorded in conjunction with some of the test sequences. For an ideal nonuniformity correction, all pixels in these images should have equal values. In practice, the pixel values have a certain standard deviation, which can be used as a performance measure of the corresponding correction algorithm. Table 7.2 shows the resulting standard deviation of the reference images for each correction method. The temporal noise standard deviation is about 3-4 units.

\begin{tabular}{|l|rr|rr|}
\hline \multirow{2}{*}{ Method } & \multicolumn{3}{|c|}{ Standard deviation } \\
\cline { 2 - 5 } & \multicolumn{2}{|c|}{ Sequence 1 } & \multicolumn{2}{c|}{ Sequence 2 } \\
& $0^{\circ} \mathrm{C}$ & $30^{\circ} \mathrm{C}$ & $0^{\circ} \mathrm{C}$ & $30^{\circ} \mathrm{C}$ \\
\hline Temporal highpass filter & 45 & 186 & 46 & 181 \\
Constant statistics & 264 & 1194 & 178 & 853 \\
MCA, offset only & 227 & 362 & 169 & 284 \\
MCA, offset and gain & 65 & 102 & 47 & 78 \\
Crossing path, offset only & 50 & 193 & 56 & 190 \\
Crossing path, offset and gain & 24 & 64 & 24 & 66 \\
\hline \hline Uncorrected reference image & 317 & 457 & 324 & 458 \\
\hline
\end{tabular}

Table 7.2: Standard deviation of uniform temperature image.

Since the fixed pattern noise is time variant, it is important that the test sequences and the reference images are related closely in time. Unfortunately, the sequences that are recorded along with the reference images are of rather poor quality. Compared to the other test sequences, the range of scene irradiance is much lower and the fixed pattern noise level is much higher. Because of this, the values in table 7.2 are probably higher than normal.

Even though the sequence quality leaves a lot to be desired, it is clear that the crossing path algorithm produces best results. When using the constant statistics algorithm, the deviation values end up very high, sometimes even higher than the standard deviation of the uncorrected image. Obviously this method requires many more input frames to deliver any useful results.

In these sequences, the average scene temperature is about $0^{\circ} \mathrm{C}$. This is one reason why the resulting deviation for the $0^{\circ} \mathrm{C}$ image is lower than for the $30^{\circ} \mathrm{C}$ image. 


\subsection{Introduced noise}

Although the correction algorithms are designed to remove fixed pattern noise, some is actually introduced as well. This is mostly caused by errors in motion estimation and image resampling. To gain some knowledge on the magnitude of this introduced noise, the nonuniformity correction algorithms are applied to a synthetic sequence of 400 frames without any temporal or fixed pattern noise. The standard deviation of the resulting offset calibration images is then used as a measure of the introduced noise level. Results for the offset-only algorithms are showed in table 7.3.

\begin{tabular}{lcc} 
& Std.dev. & Ratio \\
\hline Original sequence & 1174 & $100 \%$ \\
\hline Temporal highpass filter & 255 & $22 \%$ \\
Motion compensated average & 17 & $2 \%$ \\
Crossing path & 20 & $2 \%$
\end{tabular}

Table 7.3: Levels of introduced noise.

Not surprisingly, the level of noise introduced by the temporal highpass filter is rather high. For a longer test sequence, this value would however be lower. The other two methods have somewhat more moderate noise magnitudes.

\subsection{Synthetic sequences}

Comparing the correction algorithms is especially easy when working with synthetic sequences, where the true scene is known.

Different kinds of fixed pattern noise is added to a synthetic test sequence, an aerial view of a residential area. Sequence A features normal distributed additive noise and sine-shaped multiplicative noise. Sequence B is distorted by noise similar to that of the MSM sequences. Example frames from the two sequences are shown in figure 7.1

Nonuniformity correction is performed for 400 frames. The last corrected image is then compared to the corresponding noise free image. If the difference between the two images is regarded as noise, 


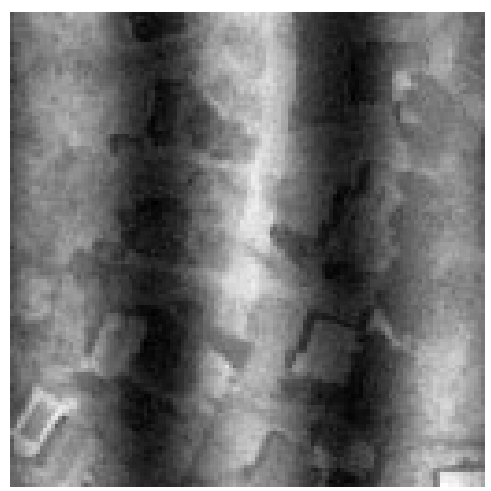

(a) Sequence A

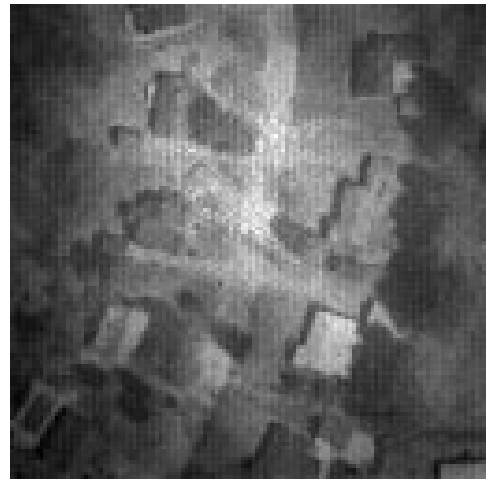

(b) Sequence B

Figure 7.1: Images from the two synthetic sequences.

the signal-to-noise ratio can be calculated as

$$
S N R=\frac{\sigma_{z}^{2}}{\sigma_{d}^{2}}
$$

where $\sigma_{z}^{2}$ is the average squared value of the noise free image and $\sigma_{d}^{2}$ is the average squared difference between the images. This is a common SNR definition that, for example, is often used in data compression to measure image degradation. Results are shown in table 7.4.

\begin{tabular}{|l|c|c|}
\hline \multirow{2}{*}{ Method } & \multicolumn{2}{|c|}{ SNR } \\
\cline { 2 - 3 } & Sequence A & Sequence B \\
\hline Temporal highpass filter & 6.4 & 6.9 \\
Constant statistics & 23.5 & 9.0 \\
MCA, offset only & 1.9 & 1.0 \\
MCA, offset and gain & 8.7 & 10.3 \\
Crossing path, offset only & 2.3 & 5.2 \\
Crossing path, offset and gain & 61.2 & 26.6 \\
\hline
\end{tabular}

Table 7.4: Signal-to-noise ratio for the sequences in figure 7.1. The higher the ratio, the more noise has been reduced.

As before, the crossing path method gives best results. Interestingly, constant statistics also gives very good results. The properties 
of the synthetic sequences are clearly in parity with the statistic assumptions. The inability to remove low-frequency shading effects results in very low signal-to-noise levels for the offset-only motion compensated average, even though most of the fixed pattern noise has been successfully removed. As a reference, figure 7.2 shows the final frame from synthetic sequence B, corrected using motion compensated average and crossing path.

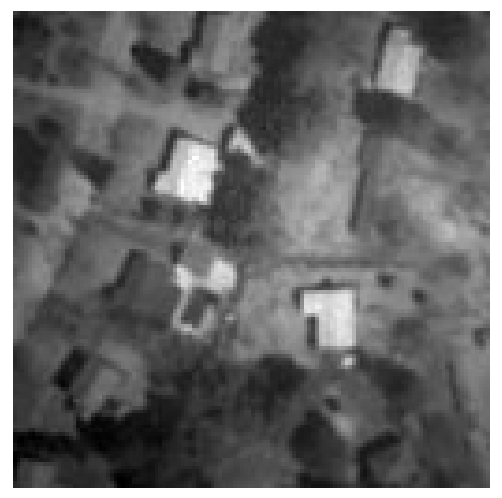

(a) Motion compensated average, offset only. $\mathrm{SNR}=1.0$

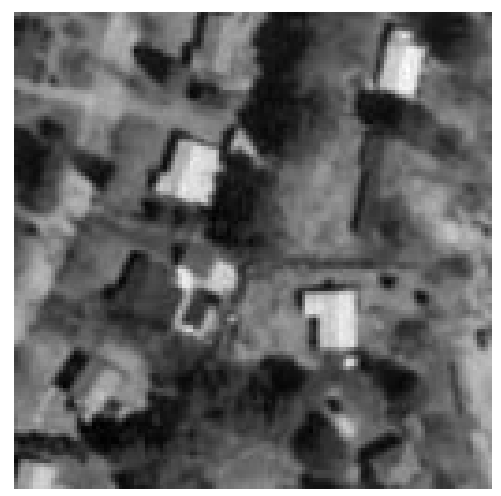

(b) Crossing path, offset and gain. $\mathrm{SNR}=26.6$

Figure 7.2: The last frame from corrected synthetic sequence B.

\subsection{Visual inspection}

As seen in the previous section, computed quality measures are not always consistent with what humans perceive as a high quality image. As a complement, the following questions are answered for each correction method when studying the sequences visually:

1. At which frame has most of the annoying high-frequency noise been removed?

2. At which frame has enough fixed pattern noise been removed so that a still image is of acceptable quality?

3. When applying the final correction parameters to the entire uncorrected sequence, how can the quality of the resulting se- 
quence be described? ( $1=$ unacceptable, $2=$ disturbing, $3=$ acceptable, $4=$ good, $5=$ excellent)

\begin{tabular}{|l|c|c|c|}
\hline Sequence & Q1 & Q2 & Q3 \\
\hline Synthetic 1 & 75 & 350 & 3 \\
MSM 1 & 150 & 350 & 3 \\
MSM 2 & 350 & - & 1 \\
\hline
\end{tabular}

(a) Temporal highpass filter

\begin{tabular}{|l|c|c|c|}
\hline Sequence & Q1 & Q2 & Q3 \\
\hline Synthetic 1 & 40 & 350 & 2 \\
MSM 1 & 150 & 150 & 4 \\
MSM 2 & 150 & 250 & 3 \\
\hline
\end{tabular}

(c) Motion compensated average, offset only

\begin{tabular}{|l|c|c|c|}
\hline Sequence & Q1 & Q2 & Q3 \\
\hline Synthetic 1 & 50 & 200 & 4 \\
MSM 1 & 80 & 80 & 3 \\
MSM 2 & 200 & 200 & 2 \\
\hline
\end{tabular}

(e) Crossing path, offset only

\begin{tabular}{|l|c|c|c|}
\hline Sequence & Q1 & Q2 & Q3 \\
\hline Synthetic 1 & 200 & 200 & 4 \\
MSM 1 & 250 & 350 & 3 \\
MSM 2 & - & - & 1 \\
\hline
\end{tabular}

(b) Constant statistics

\begin{tabular}{|l|c|c|c|}
\hline Sequence & Q1 & Q2 & Q3 \\
\hline Synthetic 1 & 40 & 150 & 2 \\
MSM 1 & 150 & 150 & 5 \\
MSM 2 & 150 & 250 & 5 \\
\hline
\end{tabular}

(d) Motion compensated average, offset and gain

\begin{tabular}{|l|c|c|c|}
\hline Sequence & Q1 & Q2 & Q3 \\
\hline Synthetic 1 & 50 & 50 & 4 \\
MSM 1 & 50 & 50 & 5 \\
MSM 2 & 180 & 180 & 4 \\
\hline
\end{tabular}

(f) Crossing path, offset and gain

Table 7.5: Test person opinions.

\section{Comments}

As can be seen for MSM sequence 2, the crossing path algorithm requires a lot more frames to converge than usual. This is because the relative translation between images is larger than one pixel for the entire first part of the sequence. The motion slows down briefly after about 150 frames, allowing for a acceptable correction in only 30 frames. This behavior also causes some artifacts in MSM sequence 1. The image motion speeds up to more than one pixel per frame just after some violations of the static scene assumptions introduce noise into the sequence, which then is left uncorrected until the motion slows down. 
The 400 frames in these sequences are barely enough for the temporal highpass filter and the constant statistics method to converge properly. Image quality would surely increase further if more frames were to be processed.

The offset-only variant of the motion compensated average method has problems with removing low-frequency shadows and variations.

\subsection{One-dimensional sensor}

As described earlier, the IRST system developed by Saab Bofors Dynamics is equipped with a scanning one-dimensional sensor array. Raw images from this system are severely disturbed by fixed pattern noise, and additional pre-processing is required to make it possible for the registration-based algorithms to detect image motion. For example, the mean values of each pixel row can be forced to be equal by adjusting detector element offset levels. This tremendously improves image quality, as can be seen in figure 7.3.

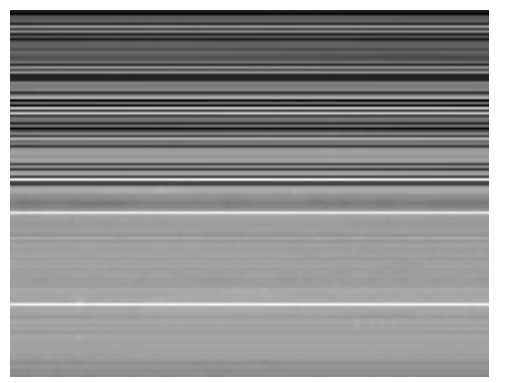

(a) Original

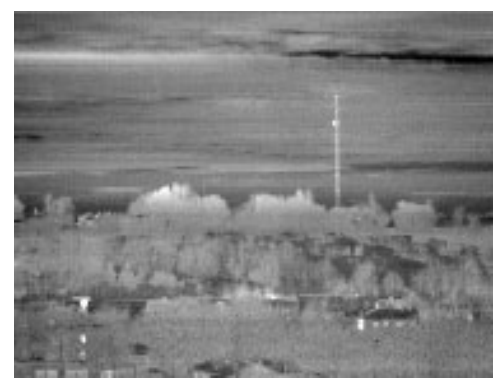

(b) Equal horizontal mean

Figure 7.3: One-dimensional sensor. Forcing the mean of each pixel row to the same value reveals the scene.

Forcing equal horizontal mean is unnecessary for the methods that do not estimate image motion. Actually, a similar procedure is carried out anyway by the temporal highpass filter, as the temporal mean is subtracted from input images, initially forcing zero mean values for all detector elements.

One might believe that this horizontal mean correction is in itself an effective calibration method. However, some disturbing artifacts 
are introduced when all rows in the image are forced to share the same mean value. For example, bright parts of the scene are followed horizontally by shadows to make the mean value constant. This can be seen for the clouds in figure 7.3(b). Furthermore, most detector elements are not corrected satisfactory with offset calibration alone, which makes horizontal stripes visible when the scene moves around.

Performing nonuniformity correction on the IRST sequences produces results similar to the MSM sequences. As usual, the temporal highpass filter and constant statistics methods introduce disturbing ghosting. Surprisingly, horizontal stripes are visible during the entire sequence for all correction methods. The sequence motion is mostly horizontal, which may explain why the registration-based methods are unable to completely suppress such noise. In fact, the stripes are least visible when using the constant statistics algorithm.

In these tests, all pixels are corrected with individual correction parameters. The fact that all pixels in a row originate from a single detector element can be exploited by using the horizontal mean value of the parameters to calibrate the entire row. This adaptation is then used in conjunction with the nonuniformity correction methods.

While the registration-based methods show little or no significant improvement, the ghosting artifacts of the temporal highpass filter and constant statistics methods are almost completely removed. As ghosting otherwise is the dominant noise component for these algorithms, image quality is significantly increased. The performance is similar to, or even better than, the registration-based algorithms. Figure 7.4 shows results for the constant statistics and crossing path methods.

With the modifications suggested here, the algorithms first produce correction parameters for a two-dimensional sensor array, which then are averaged horizontally to fit the assumptions of a one-dimensional array. It is likely that even better results can be achieved if the correction algorithms are designed for a one-dimensional sensor in the first place. This has however not been studied in this thesis. 


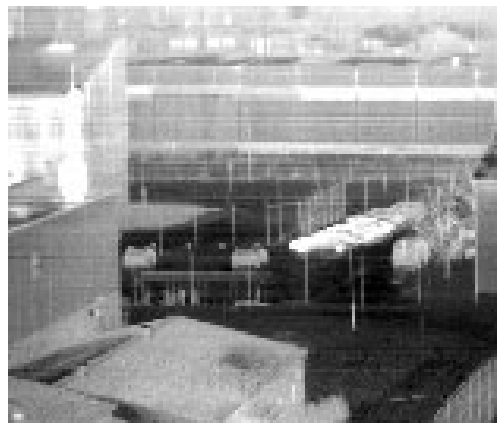

(a) Constant statistics

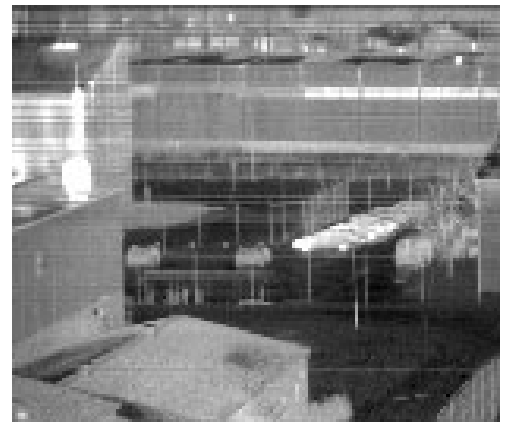

(b) Crossing path

Figure 7.4: Corrected images from a one-dimensional sensor. The constant statistics method gives equal or even better results than the more sophisticated crossing path method.

\subsection{Example results}

The following pages show frames 25, 50, 75, 100, 200 and 400 from one of the MSM test sequences, followed by the corresponding resulting images for each correction method. 


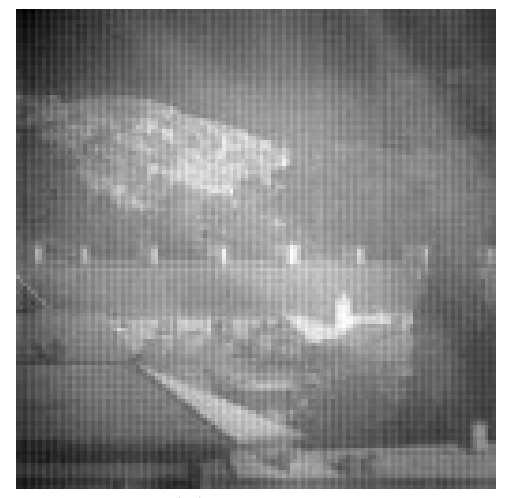

(a) Frame 25

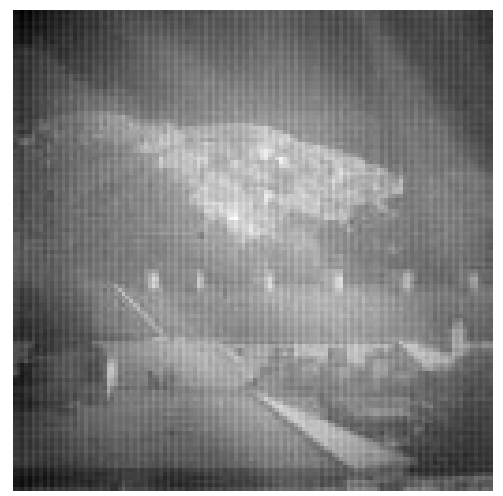

(c) Frame 75

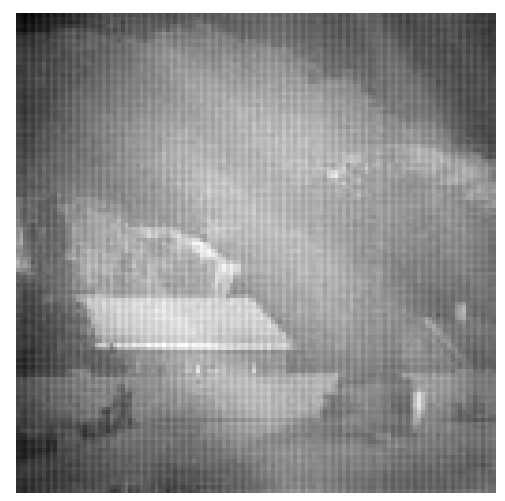

(e) Frame 200

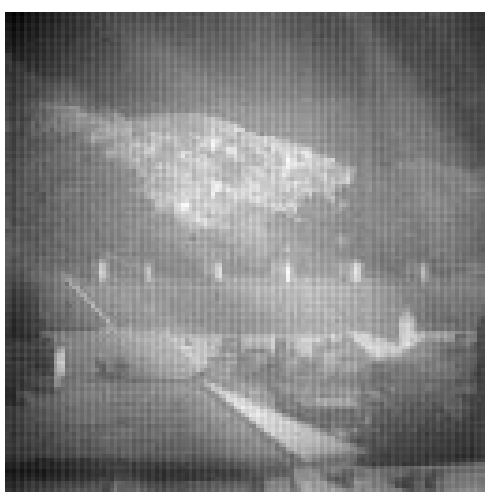

(b) Frame 50

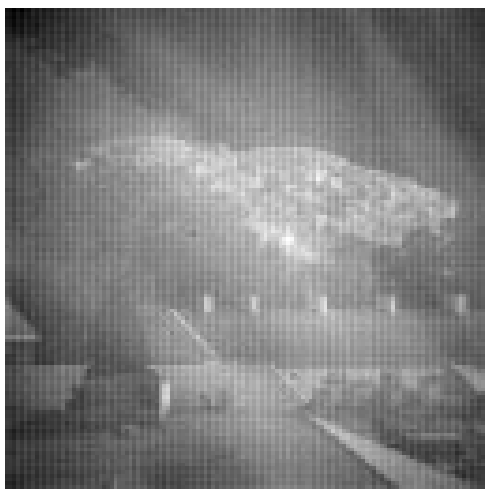

(d) Frame 100

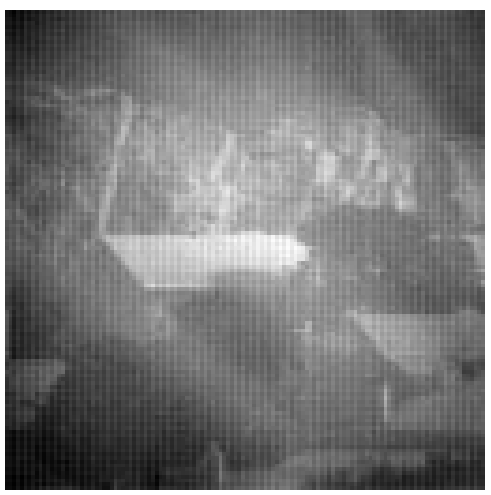

(f) Frame 400

Figure 7.5: Original sequence. 


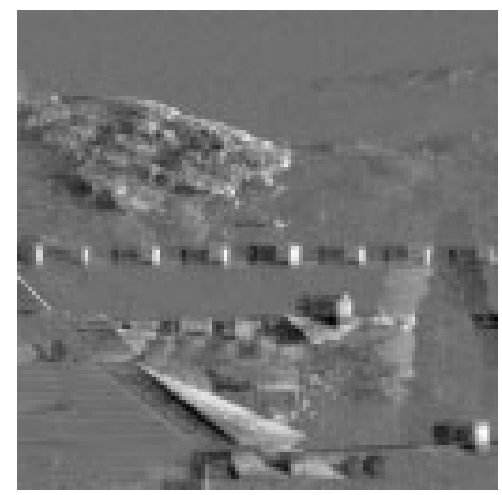

(a) Frame 25

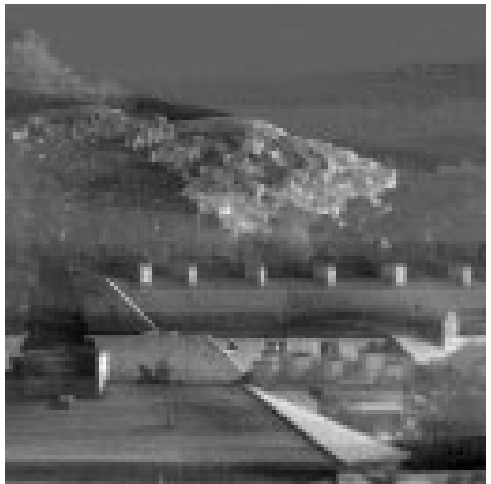

(c) Frame 75

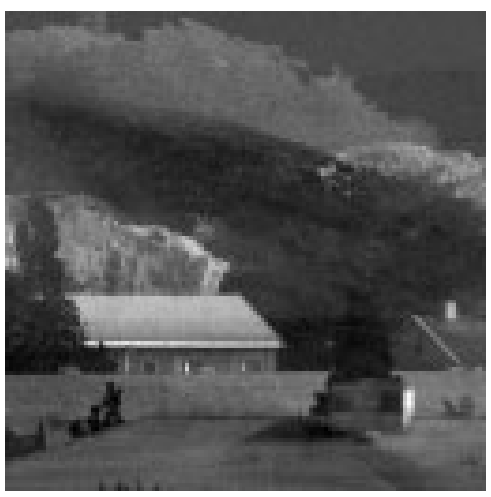

(e) Frame 200

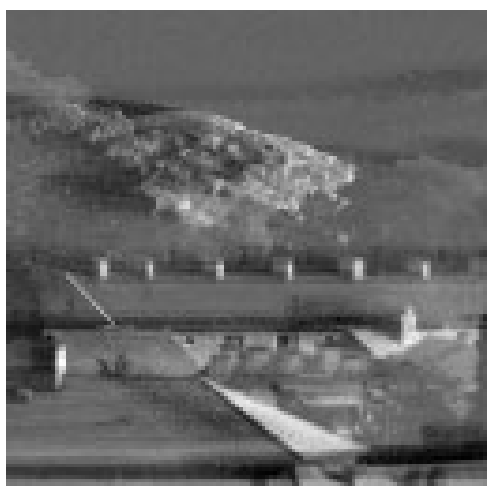

(b) Frame 50

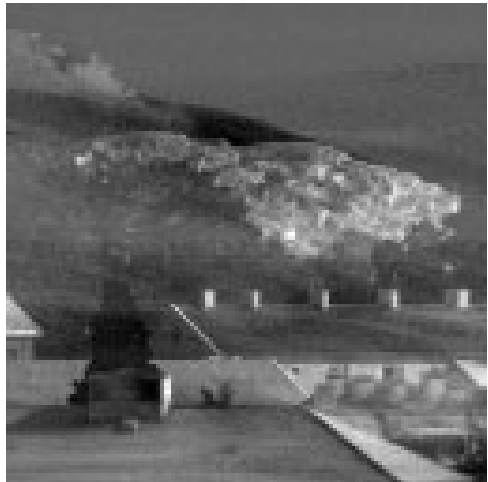

(d) Frame 100

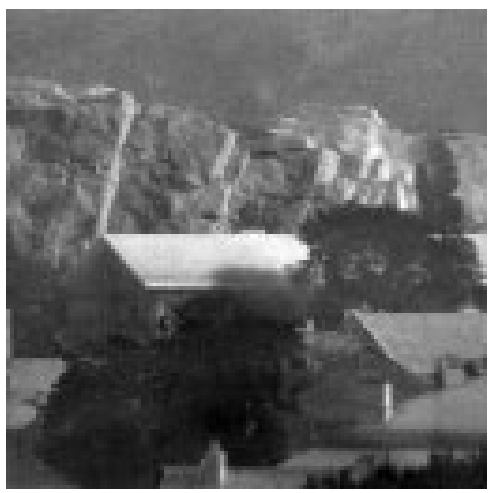

(f) Frame 400

Figure 7.6: Temporal highpass filter. 


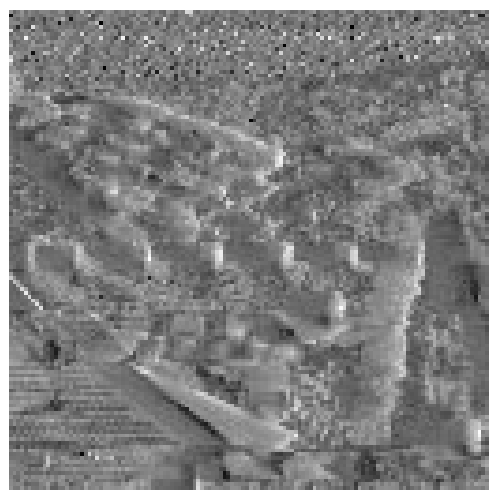

(a) Frame 25

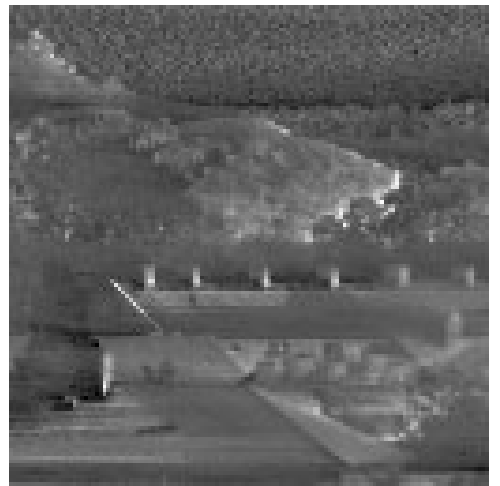

(c) Frame 75

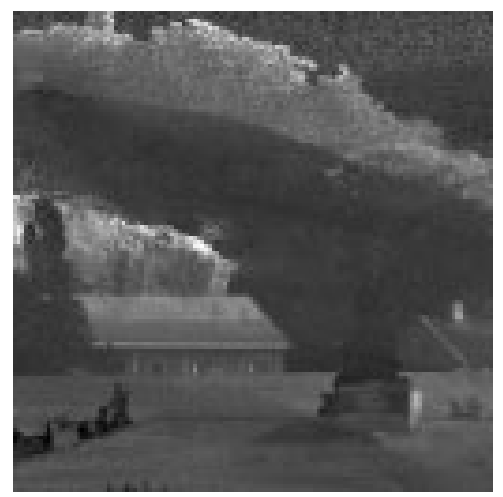

(e) Frame 200

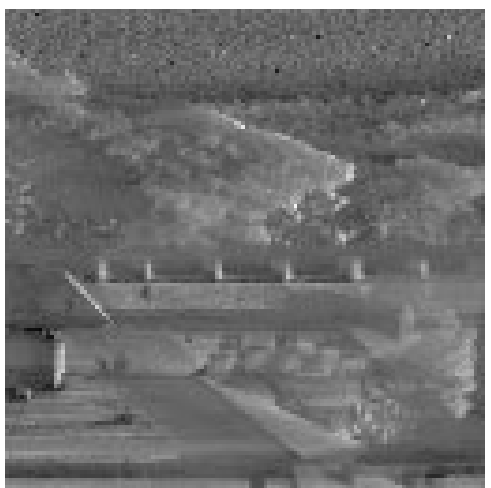

(b) Frame 50

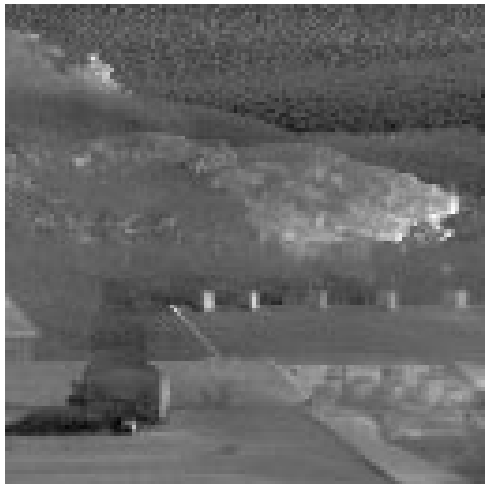

(d) Frame 100

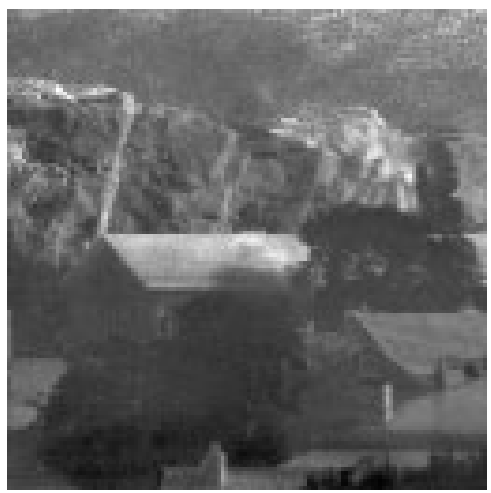

(f) Frame 400

Figure 7.7: Constant statistics. 


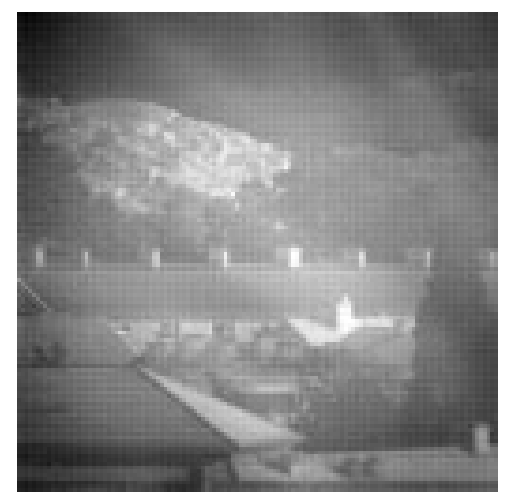

(a) Frame 25

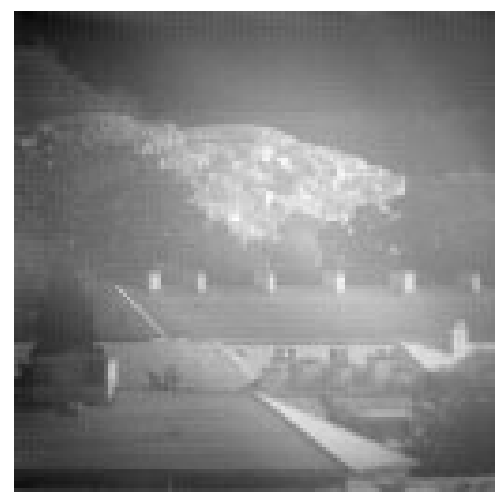

(c) Frame 75

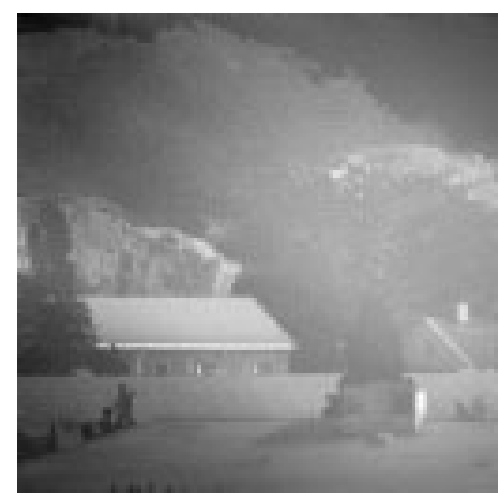

(e) Frame 200

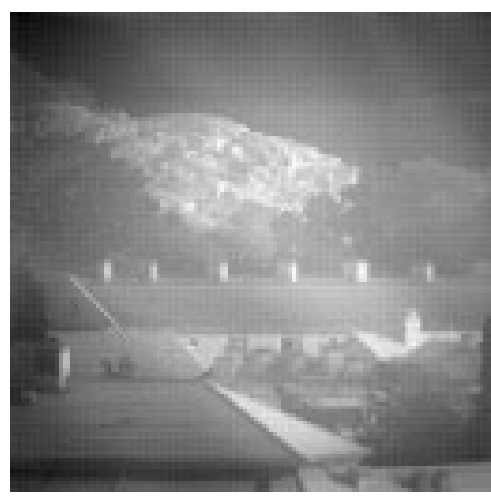

(b) Frame 50

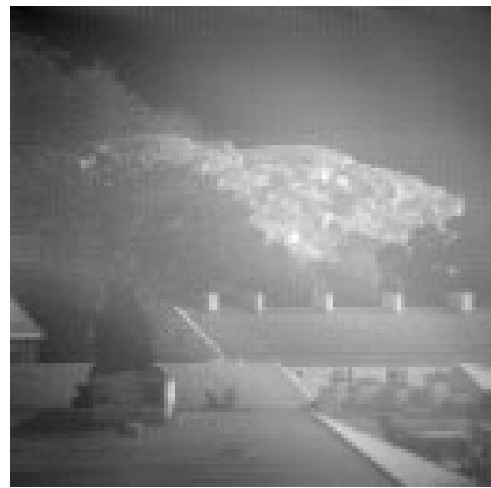

(d) Frame 100

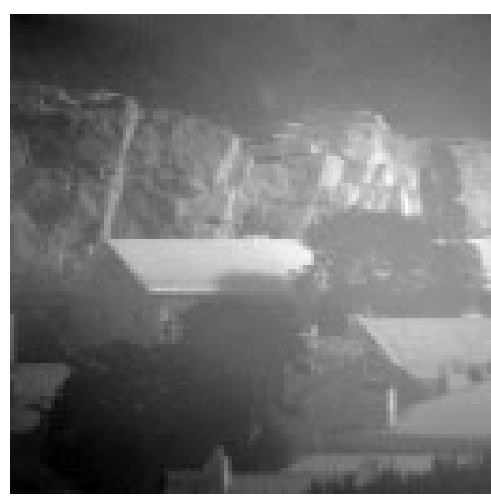

(f) Frame 400

Figure 7.8: Motion compensated average, offset only. 


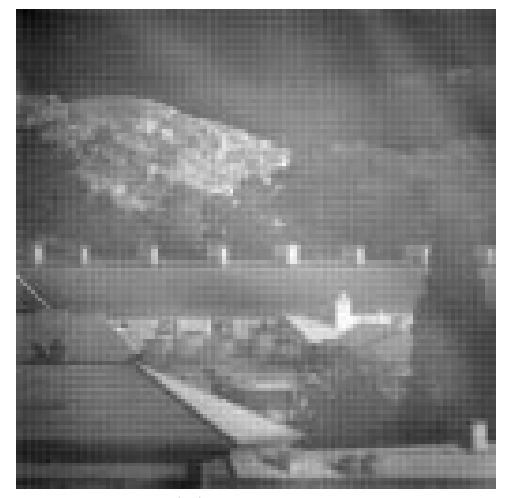

(a) Frame 25

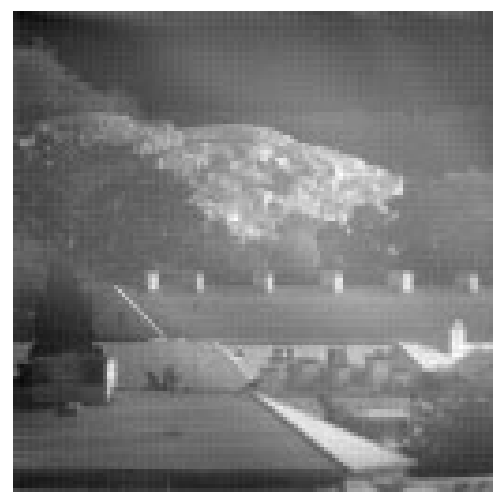

(c) Frame 75

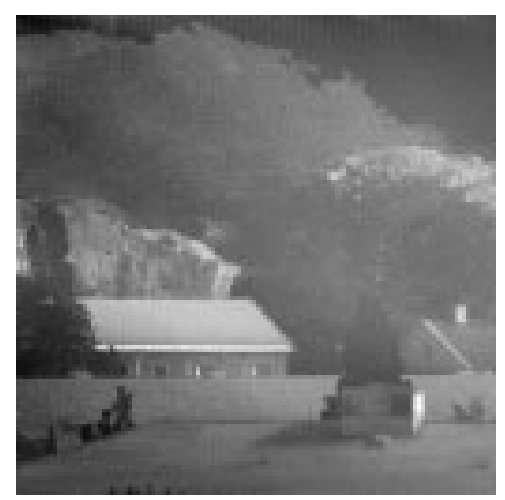

(e) Frame 200



(b) Frame 50

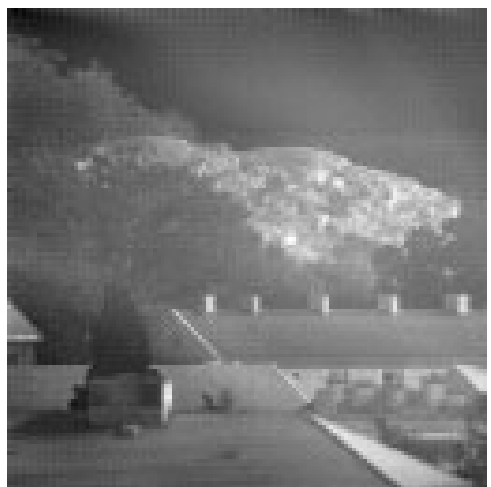

(d) Frame 100

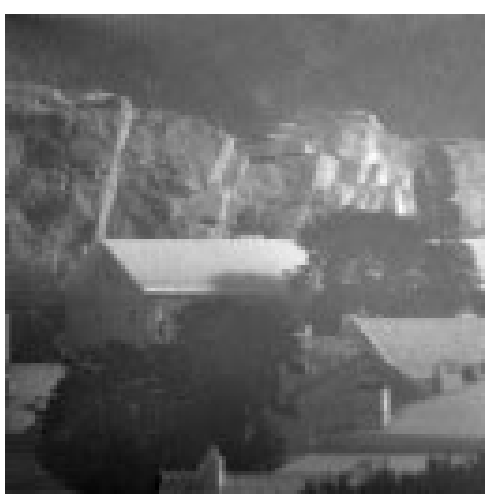

(f) Frame 400

Figure 7.9: Motion compensated average, offset and gain. 


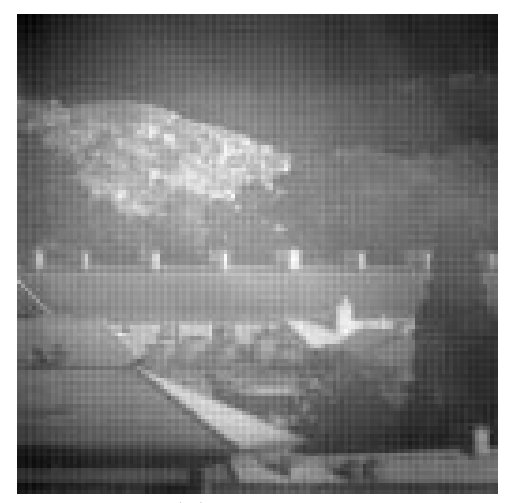

(a) Frame 25

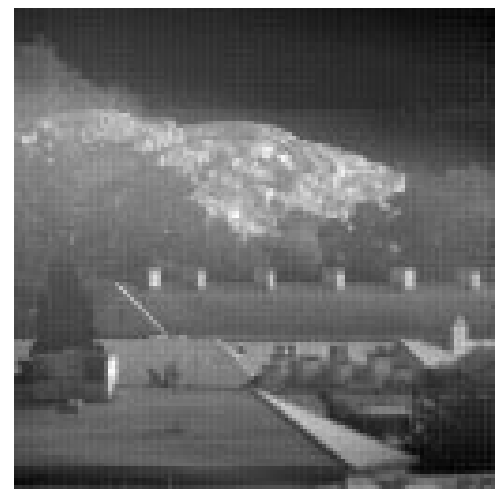

(c) Frame 75

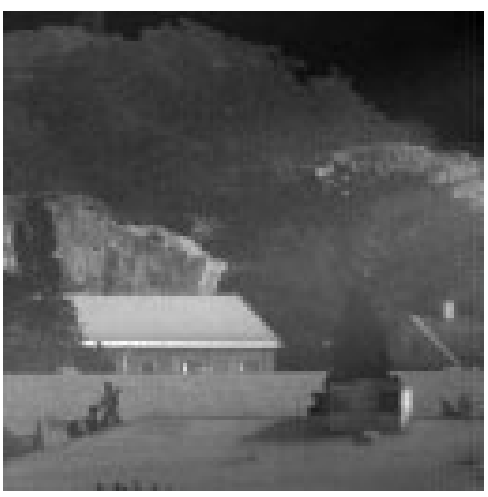

(e) Frame 200

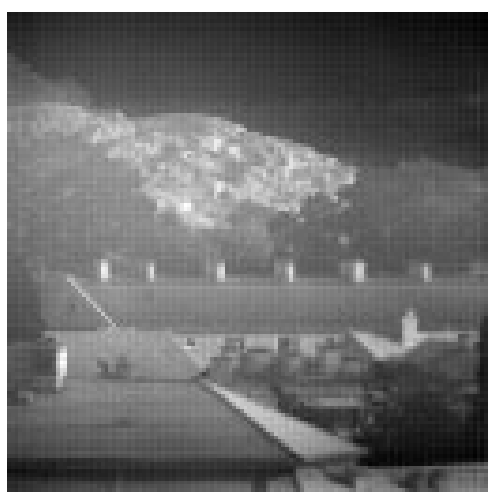

(b) Frame 50

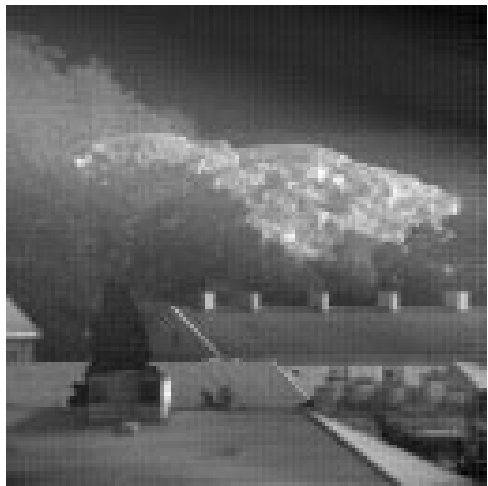

(d) Frame 100

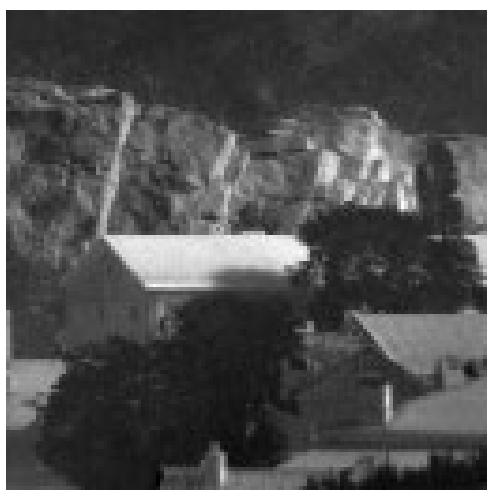

(f) Frame 400

Figure 7.10: Crossing path, offset only. 


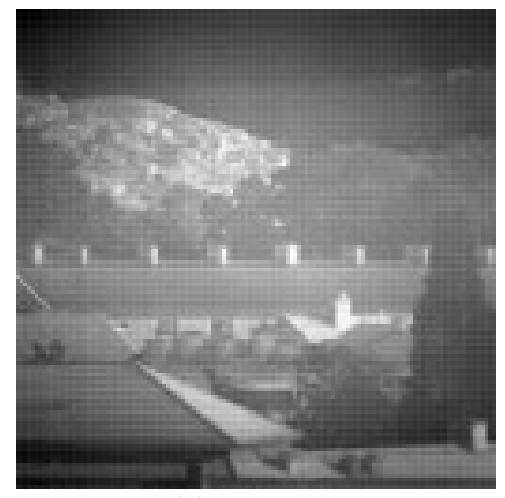

(a) Frame 25

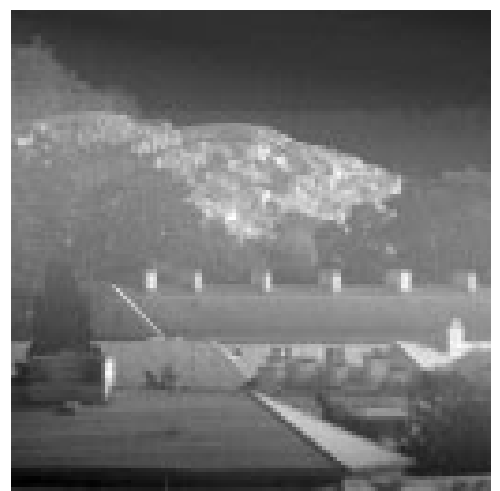

(c) Frame 75

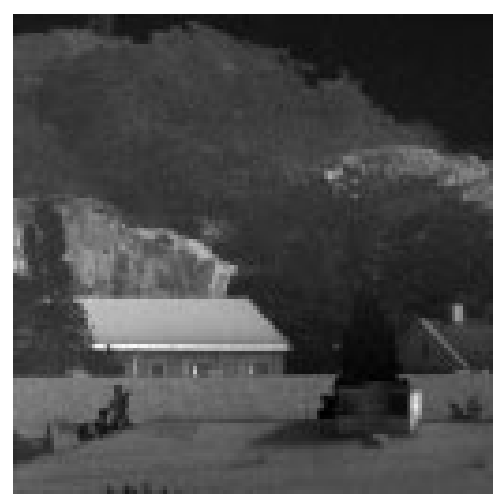

(e) Frame 200

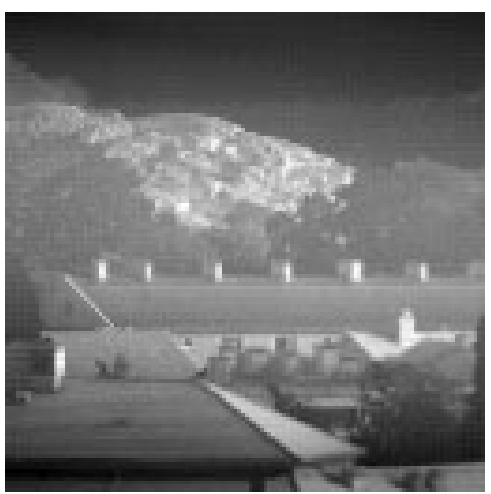

(b) Frame 50

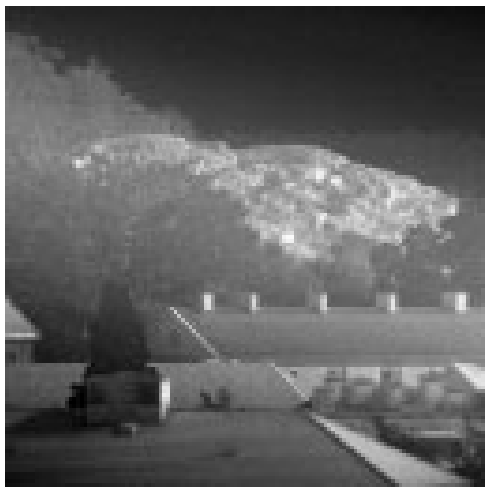

(d) Frame 100

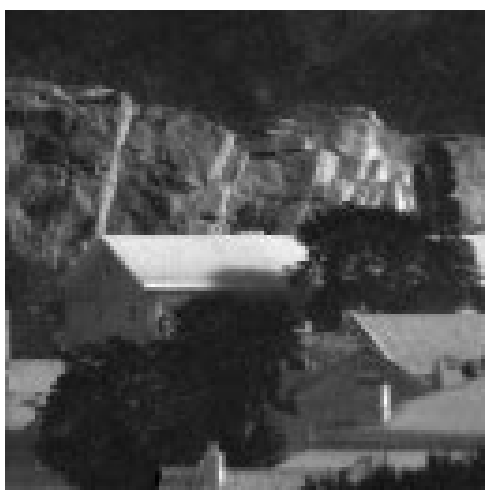

(f) Frame 400

Figure 7.11: Crossing path, offset and gain. 


\section{Chapter 8}

\section{Conclusions}

\subsection{Summary}

Several nonuniformity correction methods are described in this thesis, each with its individual pros and cons.

Temporal highpass filter and constant statistics are two well-known methods that are very easy to implement and can process sequences in real-time. Although they require many input frames before the image quality is acceptable, fixed pattern noise is usually reduced with good results. The temporal highpass filter is an offset-only algorithm, while the constant statistics method estimates both gain and offset parameters. Both methods assume that image motion exists between images in the sequence.

Crossing path, a new method that is introduced in this thesis, and motion compensated average are two somewhat more sophisticated correction methods. They are referred to as registration-based methods, in that they assume that the image motion can be accurately estimated using a motion estimation algorithm. With knowledge of the image motion, multiple detector elements that have observed the same position in the scene can be found and analyzed, leading to an efficient nonuniformity correction. In general, the registration-based methods produce very good results, but require much more computation time.

The different methods are evaluated in a number of ways using several test sequences from existing IR imaging systems, as well as synthetic data. For the special case of a scanning one-dimensional detector array, combining correction parameters from all pixels that originate from the same detector element is shown to be a way of 
significantly improving image quality.

Removing multiplicative fixed pattern noise has proven to be difficult. For both registration-based methods, the original gain parameter estimation theory was unsuccessful when put into practice and had to be modified. The difficulties are consistent with earlier experience with sensor nonuniformities at Saab Bofors Dynamics. Yet, the offset-only algorithms usually produce excellent results, suggesting that for a certain scene temperature range, the lack of multiplicative correction can be compensated by additive correction. If the scene temperature changes, disturbing artifacts would probably be visible until the algorithm stabilizes. For a fast enough method, offset-only correction might be enough for a wide temperature range. Also, each algorithm responds differently to violations of the initial assumptions, which should be taken into consideration when choosing a suitable correction method.

\subsection{Suggestions for further work}

The one-image correction procedure is an example of pre-processing that improves the image quality. The simple method presented here can surely be tweaked to perform even better, especially if it is designed for a specific sensor.

Since motion estimation is a key component of the registrationbased algorithms, research in this field is encouraged. For example, methods can be developed to detect which parts of the scene that are well suited for motion estimation. By neglecting areas of high uncertainty, estimation accuracy will improve. It would also be interesting to see what improvements can be achieved by complementing or replacing the software motion estimation with hardware position indicators on the sensor unit.

Another improvement would be to automatically detect when any basic assumptions are violated. The correction algorithm could then turn itself on and off when necessary, or at least revert uncertain parameters to an earlier state.

When correcting nonuniformity for the one-dimensional sensor, the existing algorithms are modified by averaging the correction parameters for pixels that originate from the same detector element. It would be interesting to see what improvements could be made if the algorithms were to be designed for a one-dimensional sensor in the first place. 
Finally, the correction methods described in this thesis have all been studied separately. It is possible that performance would increase if two or more methods were to be combined into one hybrid algorithm. 



\section{Bibliography}

[1] M. Törnkvist: Nonuniformity correction and property investigation of a multispectral IR camera, Diploma thesis, University of Umeå, September 1999

[2] S. Tzimopoulou, A.H. Lettington: Scene based techniques for nonuniformity correction of infrared focal plane arrays, Proc. SPIE vol. 3436, p 172-183, Infrared Technology and Applications XXIV, October 1998

[3] D.A. Scribner, K.A. Sarkady, J.T. Caulfield, M.R. Kruer, G. Katz, C.J. Gridley: Nonuniformity correction for staring IR focal plane arrays using scene-based techniques, Proc. SPIE vol. 1308, p 224-233, Infrared detectors and focal plane arrays, September 1990

[4] J.G. Harris, Y-M. Chiang: Nonuniformity correction of infrared image sequences using the constant-statistics constraint, IEEE Transactions on Image Processing, vol. 8, no. 8, p 1148-1151, August 1999

[5] J.G. Harris, Y-M. Chiang: Minimizing the ghosting artifact in scene-based nonuniformity correction, Proc. SPIE vol. 3377, $\mathrm{p}$ 106-113, Infrared Imaging Systems: Design, Analysis, Modeling and Testing IX, August 1998

[6] E. Rönnqvist: Image resolution enhancement using image sequences, M.Sc. thesis, department of electrical engineering, Linköpings universitet, May 2000.

[7] B.M. Ratliff, M.M. Hayat, R.C. Hardie: An algebraic algorithm for nonuniformity correction in focal-plane arrays, The Journal of the Optical Society of America A, vol. 19, p 1737-1747, September 2002 
[8] R.C. Hardie, M.M. Hayat, E. Armstrong, B. Yasuda: Scenebased nonuniformity correction with video sequences and registration, Applied Optics, vol. 39, no. 8, p 1241-1250, March 2000

[9] P-E. Danielsson, O. Seger, M. Magnusson Seger: Bildbehandling 2000, Image processing course litterature, Department of Systems Engineering, Linköping Institute of Technology, 2000

[10] P. Reddy, G. de Jager: Reference free nonuniformity correction for mercury cadmium telluride infrared focal plane arrays, Proc. COMSIG vol. 1998, p 243-248, 1998 


\section{På svenska}

Detta dokument hålls tillgängligt på Internet - eller dess framtida ersättare - under en längre tid från publiceringsdatum under förutsättning att inga extra-ordinära omständigheter uppstår.

Tillgång till dokumentet innebär tillstånd för var och en att läsa, ladda ner, skriva ut enstaka kopior för enskilt bruk och att använda det oförändrat för ickekommersiell forskning och för undervisning. Överföring av upphovsrätten vid en senare tidpunkt kan inte upphäva detta tillstånd. All annan användning av dokumentet kräver upphovsmannens medgivande. För att garantera äktheten, säkerheten och tillgängligheten finns det lösningar av teknisk och administrativ art.

Upphovsmannens ideella rätt innefattar rätt att bli nämnd som upphovsman i den omfattning som god sed kräver vid användning av dokumentet på ovan beskrivna sätt samt skydd mot att dokumentet ändras eller presenteras i sådan form eller i sådant sammanhang som är kränkande för upphovsmannens litterära eller konstnärliga anseende eller egenart.

För ytterligare information om Linköping University Electronic Press se förlagets hemsida http://www.ep.liu.se/

\section{In English}

The publishers will keep this document online on the Internet - or its possible replacement - for a considerable time from the date of publication barring exceptional circumstances.

The online availability of the document implies a permanent permission for anyone to read, to download, to print out single copies for your own use and to use it unchanged for any non-commercial research and educational purpose. Subsequent transfers of copyright cannot revoke this permission. All other uses of the document are conditional on the consent of the copyright owner. The publisher has taken technical and administrative measures to assure authenticity, security and accessibility.

According to intellectual property law the author has the right to be mentioned when his/her work is accessed as described above and to be protected against infringement.

For additional information about the Linköping University Electronic Press and its procedures for publication and for assurance of document integrity, please refer to its WWW home page: http://www.ep.liu.se/

\section{(C) Petter Torle}

